ESAIM: COCV 21 (2015) 465-486

DOI: $10.1051 / \mathrm{cocv} / 2014034$
ESAIM: Control, Optimisation and Calculus of Variations

www.esaim-cocv.org

\title{
A CORRECTOR FOR A WAVE PROBLEM WITH PERIODIC COEFFICIENTS IN A 1D BOUNDED DOMAIN
}

\author{
Juan Casado-Díaz ${ }^{1}$, Julio Couce-Calvo ${ }^{1}$, Faustino Maestre ${ }^{1}$ \\ AND José D. MARTín-GÓMEz ${ }^{1}$
}

\begin{abstract}
We consider a wave problem posed in a bounded open interval of $\mathbb{R}$, where the coefficients, the initial conditions and the right-hand side are highly oscillating, periodic in the space variable and almost periodic in the time one. Our purpose is to find not only the corresponding limit equation but a corrector, i.e. a strong approximation in the $H^{1}$ topology, which for the wave equation is known to be non-local. In a previous paper we have studied this problem in the whole $\mathbb{R}^{N}$, here we consider the case of a bounded domain in dimension one. Thus the novelty in this paper is the analysis of the boundary conditions.
\end{abstract}

Mathematics Subject Classification. 35B27, 35L20.

Received March 5, 2014. Revised June 12, 2014.

Published online March 4, 2015.

\section{INTRODUCTION}

The homogenization of a wave problem with oscillating coefficients in a bounded open set $\Omega \subset \mathbb{R}^{N}$ such as

$$
\left\{\begin{array}{l}
\partial_{t}\left(\rho_{\varepsilon}(x) \partial_{t} u_{\varepsilon}\right)-\operatorname{div}_{x}\left(A_{\varepsilon}(x) \nabla_{x} u_{\varepsilon}\right)=f_{\varepsilon} \text { in }(0, T) \times \Omega \\
u_{\varepsilon}=0 \text { on }(0, T) \times \partial \Omega \\
u_{\varepsilon \mid t=0}=u_{\varepsilon}^{0}, \partial_{t} u_{\varepsilon \mid t=0}=\vartheta_{\varepsilon} \quad \text { in } \Omega,
\end{array}\right.
$$

has been studied in several papers $([5,9,11])$. Assuming the coefficients $\rho_{\varepsilon}, A_{\varepsilon}$ uniformly elliptic and bounded, the right-hand side converging strongly in $L^{1}\left(0, T ; L^{2}(\Omega)\right)$ to some function $f$ and the initial data $u_{\varepsilon}^{0}, \vartheta_{\varepsilon}$ converging weakly in $H_{0}^{1}(\Omega)$ and $L^{2}(\Omega)$ respectively to some functions $u^{0}, \vartheta$, it is known that the solution $u_{\varepsilon}$ of $(1.1)$ converges in $L^{\infty}\left(0, T ; H_{0}^{1}(\Omega)\right)$ weak-* to the solution $u$ of

$$
\left\{\begin{array}{l}
\partial_{t}\left(\rho(x) \partial_{t} u\right)-\operatorname{div}_{x}\left(A(x) \nabla_{x} u\right)=f \text { in }(0, T) \times \Omega \\
u=0 \text { on }(0, T) \times \partial \Omega \\
u_{\mid t=0}=u^{0}, \partial_{t} u_{\mid t=0}=\vartheta \quad \text { in } \Omega .
\end{array}\right.
$$

Keywords and phrases. Wave equation, highly oscillating coefficients, homogenization, corrector, boundary conditions.

1 Dpto. de Ecuaciones Diferenciales y Análisis Numérico, Universidad de Sevilla, Sevilla, Spain. jcasadod@us.es; couce@us.es;

fmaestre@us.es; jdmartin@us.es 
Here the coefficient $\rho$ is the weak-* limit of $\rho_{\varepsilon}$ in $L^{\infty}(\Omega)$ and the coefficient matrix $A$ is the limit of $A_{\varepsilon}$ in the sense of the elliptic homogenization $([13,17,18])$. This homogenization result provides a weak approximation of the derivatives of $u_{\varepsilon}$. It is also interesting to get an approximation of these derivatives in the strong topology of $L^{2}((0, T) \times \Omega)$. This is called a corrector result in homogenization. Since $A$ is the limit of $A_{\varepsilon}$ in the sense of the elliptic homogenization one could expect that the elliptic corrector also provides a corrector for the wave problem. However it has been proved in [5] that this only holds true if the initial data are "well posed".

A corrector result for problem (1.1) in the case of periodic coefficients and $\Omega=\mathbb{R}^{N}$ has been obtained in [6] and [10]. For the elliptic or parabolic problems the corrector in every point is just obtained from the value of the derivative of the limit function in such point. In particular initial and boundary conditions do not affect to the corrector. However for the wave equation the corrector is non-local. In general, its value in a point depends on the value of the limit function and the right-hand side in the whole domain (or more exactly in a certain cone of dependence) and of the initial conditions. This is due to the dispersion of the waves in an heterogeneous domain. Namely, it has been considered in [10] the wave problem

$$
\left\{\begin{array}{l}
\partial_{t}\left(\rho_{\varepsilon}(t, x) \partial_{t} u_{\varepsilon}\right)-\operatorname{div}_{x}\left(A_{\varepsilon}(t, x) \nabla_{x} u_{\varepsilon}\right)+B_{\varepsilon}(t, x) \cdot \nabla_{t, x} u_{\varepsilon}=f_{\varepsilon} \quad \text { in }(0, T) \times \mathbb{R}^{N} \\
u_{\varepsilon \mid t=0}=u_{\varepsilon}^{0}, \partial_{t} u_{\varepsilon \mid t=0}=\vartheta_{\varepsilon} \quad \text { in } \mathbb{R}^{N}
\end{array}\right.
$$

where the functions $\rho_{\varepsilon}, A_{\varepsilon}, B_{\varepsilon}, f_{\varepsilon}, u_{\varepsilon}^{0}$ and $\vartheta_{\varepsilon}$ have the following structure

$$
\begin{gathered}
\rho_{\varepsilon}(t, x)=\rho^{0}\left(\frac{x}{\varepsilon}\right)+\varepsilon \rho^{1}\left(t, x, \frac{t}{\varepsilon}, \frac{x}{\varepsilon}\right), \quad A_{\varepsilon}(t, x)=A^{0}\left(\frac{x}{\varepsilon}\right)+\varepsilon A^{1}\left(t, x, \frac{t}{\varepsilon}, \frac{x}{\varepsilon}\right), \quad B_{\varepsilon}(t, x)=B\left(t, x, \frac{t}{\varepsilon}, \frac{x}{\varepsilon}\right) \\
f_{\varepsilon}(t, x)=f\left(t, x, \frac{t}{\varepsilon}, \frac{x}{\varepsilon}\right), \quad u_{\varepsilon}^{0}(x)=u^{0}(x)+\varepsilon u^{1}\left(x, \frac{x}{\varepsilon}\right), \quad \vartheta_{\varepsilon}(x)=\vartheta\left(x, \frac{x}{\varepsilon}\right),
\end{gathered}
$$

and they are periodic in $y=x / \varepsilon$ of period the unitary cube $Y$ in $\mathbb{R}^{N}$ and almost-periodic in $s=t / \varepsilon$. Then it has been proved the corrector result

$$
u_{\varepsilon}(t, x)-u_{0}(t, x)-\varepsilon u_{1}\left(t, x, \frac{t}{\varepsilon}, \frac{x}{\varepsilon}\right) \rightarrow 0 \text { in } H^{1}\left((0, T) \times \mathbb{R}^{N}\right),
$$

where $u_{0}, u_{1}$ (and some $u_{2}$ ) solve

$$
\left\{\begin{array}{l}
\rho^{0} \partial_{s s}^{2} u_{1}-\operatorname{div}_{y}\left(A^{0}\left(\nabla_{x} u_{0}+\nabla_{y} u_{1}\right)\right)=0 \\
\rho^{0} \partial_{t t}^{2} u_{0}-\operatorname{div}_{x}\left(A^{0} \nabla_{x} u_{0}\right)+2 \rho^{0} \partial_{s t}^{2} u_{1}-\operatorname{div}_{x}\left(A^{0} \nabla_{y} u_{1}\right)-\operatorname{div}_{y}\left(A^{0} \nabla_{x} u_{1}\right) \\
+\partial_{s}\left(\rho^{1}\left(\partial_{t} u_{0}+\partial_{s} u_{1}\right)\right)-\operatorname{div}_{y}\left(A^{1}\left(\nabla_{x} u_{0}+\nabla_{y} u_{1}\right)\right) \\
+B \cdot\left(\nabla_{t, x} u_{0}+\nabla_{s, y} u_{1}\right)+\rho^{0} \partial_{s s}^{2} u_{2}-\operatorname{div}_{y}\left(A^{0} \nabla_{y} u_{2}\right)=f \\
u_{1}(t, x, s, y), u_{2}(t, x, s, y) \text { periodic of period } Y \text { in } y \text { and almost periodic in } s \\
u_{0 \mid t=0}=u^{0}, \quad\left(\partial_{t} u_{0}+\partial_{s} u_{1}\right)_{\mid t=s=0}=\vartheta, \quad \partial_{y} u_{1} \mid t=s=0 \\
=\partial_{y} u^{1} .
\end{array}\right.
$$

Here the second equation is just formal. The corresponding variational formulation consider test functions $\psi=\psi(t, x, s, y)$ satisfying the wave equation

$$
\rho^{0} \partial_{s s}^{2} \psi-\operatorname{div}_{y}\left(A^{0} \nabla_{y} \psi\right)=0,
$$

and then the terms containing $u_{2}$ disappear. Therefore, in this formulation we get a system of two equations for the two functions $u_{0}, u_{1}$ which appear in (1.3).

We observe that the second equation in (1.4) contains derivatives of $u_{1}$ not only in the microscopic variables $(s, y)$ but also in the macroscopic ones $(t, x)$. This is completely different to the elliptic and parabolic cases and 
as we mentioned above shows that the corrector is non-local for the wave equation. The behavior of $u_{1}$ in a point $(t, x, s, y)$ does not only depend on the value of $u_{0}$ in $(t, x)$. As a consequence of this non-local behavior, it is proved in [10] that the presence of the first order term $B_{\varepsilon}(t, x) \cdot \nabla u_{\varepsilon}$ in (1.2) provides a non-local problem for the limit $u_{0}$ of $u_{\varepsilon}$. On the other hand, we observe that due to the presence of derivatives in $t$ for $u_{1}$ in the second equation of (1.4) we have needed to introduce initial conditions for $u_{1}$ in $t=0$.

An interesting question is if a result like (1.3) holds also true for a bounded domain $\Omega$. In such case a formal calculus shows that $u_{0}, u_{1}$ must also satisfy (1.4) where now it would be necessary to introduce some boundary conditions for $u_{1}$ on $\partial \Omega$. These boundary conditions must depend on the boundary conditions imposed for $u_{\varepsilon}$ in (1.2) and probably on the geometry of $\Omega$. For this reason the problem in a bounded domain seems to be much more difficult than the problem in $\mathbb{R}^{N}$. In the present paper we analyze this question in the simplest case where $\Omega$ is a bounded one-dimensional interval $(\alpha, \beta)$. Our formulation considers Dirichlet, Neumann and mixed boundary conditions. We show that (1.3) holds still true, but just for a subsequence because the behavior of the derivatives of $u_{\varepsilon}$ depends on the relative position of the extremes of the interval $(\alpha, \beta)$ with respect to the periodicity cell. The main difficulty is to find the good boundary conditions for $u_{1}$. They describe the shocks of the wave with the walls. Namely, we show that $u_{1}$ can be decomposed as

$$
u_{1}(t, x, s, y)=\hat{u}_{1}(t, x, y)+\sum_{j \in J_{1}} \tilde{\mathrm{u}}_{j}(t, x) \Phi_{j}(y) \mathrm{e}^{\mathrm{i} \lambda_{j} s}+\sum_{j \in J_{2}}\left(\tilde{\mathrm{u}}_{j 1}(t, x) \Phi_{j 1}(y)+\tilde{\mathrm{u}}_{j 2}(t, x) \Phi_{j 2}(y)\right) \mathrm{e}^{\mathrm{i} \lambda_{j} s}
$$

where $\hat{u}_{1}$ is the classical elliptic corrector, the numbers $\lambda_{j}$ are the (negative and positive) square roots of the positive eigenvalues $\mu_{j}$ of the following problem, with $a^{0}$ the above function $A^{0}$ which is now denoted by a lowercase letter to emphasize that it is a scalar function.

$$
\left\{\begin{array}{l}
-\frac{\mathrm{d}}{\mathrm{d} y}\left(a^{0} \frac{\mathrm{d} \Phi}{\mathrm{d} y}\right)=\mu_{j} \rho^{0} \Phi \text { in } \mathbb{R} \\
\Phi, a^{0} \frac{\mathrm{d} \Phi}{\mathrm{d} y} \text { periodic of period } Y .
\end{array}\right.
$$

The sets $J_{1}$ and $J_{2}$ correspond to the index $j$ such that $\mu_{j}$ has multiplicity one or two respectively and the functions $\Phi_{j}, \Phi_{j 1}, \Phi_{j 2}$ are a basis of the corresponding eigenfunctions spaces. The boundary conditions for $u_{1}$ are in fact given for the functions $\tilde{u}_{j 1}, \tilde{u}_{j 2}$ associated to the eigenvalues of multiplicity two while boundary conditions for the functions $\tilde{u}_{j}$ are not needed. This different behavior corresponds to the fact that the perturbations associated to simple eigenvalues do not propagate in space while the corresponding to eigenvalues of multiplicity two travel in space.

We observe that (1.5) corresponds to the eigenvalue problem (2) in $[6]$ for $l=0$, where a corrector for problem (1.1) with $\Omega=\mathbb{R}^{N}$ and coefficients periodic of period $\varepsilon$ is obtained by using the Bloch theory. This is not surprising because we are studying very related problems but in our case this is the only eigenvalue problem we need, while in $[6]$ it is necessary to introduce the second parameter $l \in \mathbb{Z}^{N}$.

\section{NOTATION}

The functions will be assumed valued in the complex field $\mathbb{C}$, with $i$ the imaginary unit. The conjugate of a vector $u \in \mathbb{C}^{k}$ is denoted by $\bar{u}$. The real part of a complex number $z$ is denoted by $\operatorname{Re}(z)$.

In order to write shorter expressions, we will only write the arguments of the functions when it is essential.

For $\alpha, \beta \in \mathbb{R}, \alpha<\beta$, we denote by $I$ the interval $I=(\alpha, \beta) \subset \mathbb{R}$.

For $T>0$, we denote by $Q_{T}$ the open set $(0, T) \times I$.

We denote by $O_{\varepsilon}$ an arbitrary sequence which converges to zero when $\varepsilon$ tends to zero and which can change from line to line.

We denote by $Y$ the unitary interval $(0,1)$. 
For functions defined in $Y$, we use the index $\sharp$ to note periodicity with respect to $Y$ (thus the functions are in fact defined in the whole of $\mathbb{R})$. For example $L_{\sharp}^{1}(Y)$ denotes the space of functions in $L_{\text {loc }}^{1}(\mathbb{R})$ which are periodic of period $Y$. The integral in $Y$ of a function $u$ in $L_{\sharp}^{1}(Y)$ will be denoted by $M_{y}(u)$ (mean value).

For functions defined in $\mathbb{R}$, the index $\sharp$ denotes almost-periodicity. Namely, we will use the following spaces of almost-periodic functions.

1. We denote by $C_{\sharp}(\mathbb{R})$ the space of almost-periodic functions in the Bohr sense, i.e. the closure of the trigonometric polynomials $u$ of the form

$$
u(s)=\sum_{j=1}^{m} c_{p_{j}} \mathrm{e}^{\mathrm{i} p_{j} s}, \quad \forall s \in \mathbb{R}, \quad \text { with } m \in \mathbb{N}, p_{j} \in \mathbb{R}, c_{p_{j}} \in \mathbb{C}, 1 \leq j \leq m,
$$

with respect to the uniform convergence topology.

2. We denote by $L_{\sharp}^{p}(\mathbb{R}), 1 \leq p<+\infty$ the space of Besicovitch defined as the closure of the trigonometric polynomials as above with respect to the norm

$$
\|u\|_{L_{\sharp}^{p}(\mathbb{R})}=\left(\lim _{R \rightarrow \infty} \frac{1}{2 R} \int_{-R}^{R}|u(s)|^{p} \mathrm{~d} s\right)^{\frac{1}{p}} .
$$

Indeed, $\|\cdot\|_{L_{\sharp}^{p}(\mathbb{R})}$ is not a norm but a seminorm. In order to have a structure of normed space in $L_{\sharp}^{p}(\mathbb{R})$ it is necessary to work with a quotient space, i.e. to identify functions $u, v \in L_{\sharp}^{p}(\mathbb{R})$ such that $\|u-v\|_{L_{\sharp}^{p}(\mathbb{R})}=0$. It is well known that every function $u$ in the space $L_{\sharp}^{1}(\mathbb{R})$ has a mean value $M_{s}(u) \in \mathbb{C}$, which is defined by

$$
\lim _{\varepsilon \rightarrow 0} \int_{E} u\left(\frac{s}{\varepsilon}\right) \mathrm{d} s=M_{s}(u)|E|, \quad \forall E \subset \mathbb{R}, \quad \text { bounded and measurable. }
$$

3. We denote by $H_{\sharp}^{k}(\mathbb{R}), k \in \mathbb{R}$ (in the paper we just use $k=-1,0,1$ ) by

$$
H_{\sharp}^{k}(\mathbb{R})=\left\{\sum_{p \in \mathbb{R}} c_{p} e^{\mathrm{i} p s}: c_{p} \in \mathbb{C}, \forall p \in \mathbb{R}, \sum_{p \in \mathbb{R} \backslash\{0\}}\left|c_{p}\right|^{2}|p|^{2 k}<\infty\right\} .
$$

It is a Hilbert space endowed with the norm

$$
\left\|\sum_{p \in \mathbb{R}} c_{p} e^{\mathrm{i} p s}\right\|_{H_{\sharp}^{k}(\mathbb{R})}=\left(\left|c_{0}\right|^{2}+\sum_{p \in \mathbb{R} \backslash\{0\}}\left|c_{p}\right|^{2}|p|^{2 k}\right)^{\frac{1}{2}} .
$$

We remark that $H_{\sharp}^{0}(\mathbb{R})=L_{\sharp}^{2}(\mathbb{R})$. Moreover, we have $H_{\sharp}^{k}(\mathbb{R})^{\prime}=H_{\sharp}^{-k}(\mathbb{R})$.

In $H_{\sharp}^{k}(\mathbb{R})$ we define the derivative operator $\frac{\mathrm{d}}{\mathrm{d} s}: H_{\sharp}^{k}(\mathbb{R}) \rightarrow H_{\sharp}^{k-1}(\mathbb{R})$ just by

$$
\frac{\mathrm{d}}{\mathrm{d} s}\left(\sum_{p \in \mathbb{R}} c_{p} e^{\mathrm{i} p s}\right)=\sum_{p \in \mathbb{R}} i p c_{p} e^{\mathrm{i} p s} .
$$

We will also use the index $\sharp$ for functions defined in $\mathbb{R} \times Y$ to denote periodicity with respect to $Y$ and almost periodicity with respect to $\mathbb{R}$. Namely, we will work with the spaces $C_{\sharp}(\mathbb{R} \times Y), L_{\sharp}^{p}(\mathbb{R} \times Y), H_{\sharp}^{k}(\mathbb{R} \times Y)$, which are constructed as above but starting from functions of the form

$$
\begin{aligned}
& u(s, y)=\sum_{j=1}^{m} c_{p_{j} n_{j}} \mathrm{e}^{\mathrm{i}\left(p_{j} s+2 \pi n_{j} y\right)}, \quad \forall(s, y) \in \mathbb{R} \times Y, \\
& m \in \mathbb{N}, p_{j} \in \mathbb{R}, n_{j} \in \mathbb{Z}, c_{p_{j} n_{j}} \in \mathbb{C}, \quad 1 \leq j \leq m .
\end{aligned}
$$

The mean value of a function $u$ in $L_{\sharp}^{1}(\mathbb{R} \times Y)$ will be denoted by $M_{s, y}(u)=M_{s}\left(M_{y}(u)\right)=M_{y}\left(M_{s}(u)\right)$. 
Along the paper we will consider two fixed periodic real functions $\rho^{0} \in L_{\sharp}^{\infty}(Y)$ and $a^{0} \in L_{\sharp}^{\infty}(Y)$, such that

$$
\underset{y \in Y}{\operatorname{infess}} \rho^{0}(y)>0, \quad \operatorname{infess}_{y \in Y} a^{0}(y)>0 .
$$

Then, we introduce the spaces $\mathcal{W}^{k}, k \in \mathbb{R}$ by

$$
\mathcal{W}^{k}=\left\{\sum_{p \in \mathbb{R}} c_{p}(y) \mathrm{e}^{\mathrm{i} p s} \in H_{\sharp}^{k}(\mathbb{R} \times Y): c_{0} \equiv 0, \quad \rho^{0} \partial_{s s}^{2} w-\partial_{y}\left(a^{0} \partial_{y} w\right)=0\right\} .
$$

A spectral decomposition for the elements of $\mathcal{W}^{k}$ can be obtained in the following way: we introduce $\mu_{0}=0<$ $\mu_{1}<\mu_{2}<\cdots$ as the numbers (eigenvalues) $\mu_{j} \in[0,+\infty)$ satisfying that the space

$$
W_{j}=\left\{\Phi \in H_{\sharp}^{1}(Y):-\frac{\mathrm{d}}{\mathrm{d} y}\left(a^{0} \frac{\mathrm{d} \Phi}{\mathrm{d} y}\right)=\mu_{j} \rho^{0} \Phi \text { in } \mathbb{R}\right\}
$$

does not reduce to the null space. For an eigenvalue $\mu_{j}$, we denote

$$
W_{-j}=W_{j}, \quad \lambda_{j}=\sqrt{\mu_{j}}, \quad \lambda_{-j}=-\sqrt{\mu_{j}}
$$

Then,

$$
\mathcal{W}^{k}=\left\{\sum_{\substack{j=-\infty \\ j \neq 0}}^{\infty} c_{j}(y) \mathrm{e}^{\mathrm{i} \lambda_{j} s}: c_{j} \in W_{j}, \forall j \in \mathbb{Z}, \sum_{\substack{j=-\infty \\ j \neq 0}}^{\infty}\left\|c_{j}\right\|_{L_{\sharp}^{2}(Y)}^{2}\left|\lambda_{j}\right|^{2 k}<\infty\right\} .
$$

We remark that with our definitions, the space $H_{\sharp}^{1}(\mathbb{R} \times Y)$ is not contained in $L_{\sharp}^{2}(\mathbb{R} \times Y)$ since

$$
\sum_{p \in \mathbb{R} \backslash\{0\}}\left\|c_{p}\right\|_{L_{\sharp}^{2}(Y)}^{2}|p|^{2}<\infty \nRightarrow \sum_{p \in \mathbb{R} \backslash\{0\}}\left\|c_{p}\right\|_{L_{\sharp}^{2}(Y)}^{2}<\infty,
$$

but $\mathcal{W}^{1}$ is contained in $\mathcal{W}^{0}$ because

$$
\sum_{\substack{j=-\infty \\ j \neq 0}}^{\infty}\left\|c_{j}\right\|_{L_{\sharp}^{2}(Y)}^{2} \leq \frac{1}{\lambda_{1}^{2}} \sum_{\substack{j=-\infty \\ j \neq 0}}^{\infty}\left\|c_{j}\right\|_{L_{\sharp}^{2}(Y)}^{2}\left|\lambda_{j}\right|^{2} .
$$

We also recall the following well known result: If $\Phi^{1}, \Phi^{2}$ are two solutions of the differential equation

$$
-\frac{\mathrm{d}}{\mathrm{d} y}\left(a^{0} \frac{\mathrm{d} \Phi}{\mathrm{d} y}\right)=\mu_{j} \rho^{0} \Phi \text { in } \mathbb{R}
$$

then

$$
a^{0}\left(\Phi^{1} \frac{\mathrm{d} \Phi^{2}}{\mathrm{~d} y}-\Phi^{2} \frac{\mathrm{d} \Phi^{1}}{\mathrm{~d} y}\right) \text { is constant in } \mathbb{R}
$$

where the constant is zero if and only if $\Phi^{1}, \Phi^{2}$ are linearly dependent.

Taking into account that the spaces $W_{j}$ can be of dimension one or two, we denote

$$
J_{1}=\left\{j \in \mathbb{Z} \backslash\{0\}: \operatorname{dim}\left(W_{j}\right)=1\right\}, \quad J_{2}=\left\{j \in \mathbb{Z} \backslash\{0\}: \operatorname{dim}\left(W_{j}\right)=2\right\},
$$

and

$$
J_{1}^{+}=J_{1} \cap \mathbb{Z}^{+}, \quad J_{2}^{+}=J_{2} \cap \mathbb{Z}^{+}
$$


For a function

$$
u=\sum_{\substack{j=-\infty \\ j \neq 0}}^{\infty} c_{j}(y) \mathrm{e}^{\mathrm{i} \lambda_{j} s} \in \mathcal{W}^{0},
$$

we define $P_{1} u, P_{2} u$ by

$$
P_{1} u=\sum_{j \in J_{1}} c_{j}(y) \mathrm{e}^{\mathrm{i} \lambda_{j} s}, \quad P_{2} u=\sum_{j \in J_{2}} c_{j}(y) \mathrm{e}^{\mathrm{i} \lambda_{j} s} .
$$

Besides of the functions $\rho^{0}, a^{0}$, we will also consider functions $\rho^{1} \in L^{\infty}\left(Q_{T} ; C_{\sharp}(\mathbb{R} \times Y)\right), a^{1} \in L^{\infty}\left(Q_{T} ; C_{\sharp}(\mathbb{R} \times\right.$ $Y)), \rho^{1}, a^{1}$ real, such that

$$
\partial_{t} \rho^{1}, \partial_{s} \rho^{1}, \partial_{t} a^{1}, \partial_{s} a^{1} \in L^{\infty}\left(Q_{T} ; C_{\sharp}(\mathbb{R} \times Y)\right),
$$

and a function $B \in L^{\infty}\left(Q_{T} ; C_{\sharp}(\mathbb{R} \times Y)\right)^{2}$.

With these functions, and $\varepsilon>0$, we will define $\rho_{\varepsilon}, a_{\varepsilon} \in W^{1, \infty}\left(0, T ; L^{\infty}(I)\right), B_{\varepsilon} \in L^{\infty}\left(Q_{T}\right)^{2}$ by

$$
\begin{gathered}
a_{\varepsilon}(t, x)=a^{0}\left(\frac{x}{\varepsilon}\right)+\varepsilon a^{1}\left(t, x, \frac{t}{\varepsilon}, \frac{x}{\varepsilon}\right) \\
\rho_{\varepsilon}(t, x)=\rho^{0}\left(\frac{x}{\varepsilon}\right)+\varepsilon \rho^{1}\left(t, x, \frac{t}{\varepsilon}, \frac{x}{\varepsilon}\right) \\
B_{\varepsilon}(t, x)=B\left(t, x, \frac{t}{\varepsilon}, \frac{x}{\varepsilon}\right),
\end{gathered}
$$

a.e. $(t, x) \in Q_{T}$.

We denote by $c_{\alpha}, d_{\alpha}, c_{\beta}, d_{\beta}$ complex constants such that

$$
\left|c_{\alpha}\right|+\left|d_{\alpha}\right|>0, \quad\left|c_{\beta}\right|+\left|d_{\beta}\right|>0 .
$$

With these constants, we define the space $V$ by

$$
V=\left\{v \in H^{1}(I): v(\alpha)=0 \text { if } c_{\alpha}=0 \text { and } v(\beta)=0 \text { if } c_{\beta}=0\right\} .
$$

The space $V$ is endowed with the $H^{1}(I)$ norm.

\section{MAin RESUlts}

For $u_{\varepsilon}^{0}, \vartheta_{\varepsilon}$ and $f_{\varepsilon}$ bounded in $V, L^{2}(I)$ and $L^{2}\left(Q_{T}\right)$ respectively, we consider the wave problem

$$
\left\{\begin{array}{l}
\partial_{t}\left(\rho_{\varepsilon} \partial_{t} u_{\varepsilon}\right)-\partial_{x}\left(a_{\varepsilon} \partial_{x} u_{\varepsilon}\right)+B_{\varepsilon} \cdot \nabla_{t, x} u_{\varepsilon}=f_{\varepsilon} \text { in } Q_{T} \\
\left(-c_{\alpha} a_{\varepsilon} \partial_{x} u_{\varepsilon}+d_{\alpha} u_{\varepsilon}\right)_{\mid x=\alpha}=0,\left(c_{\beta} a_{\varepsilon} \partial_{x} u_{\varepsilon}+d_{\beta} u_{\varepsilon}\right)_{\mid x=\beta}=0 \text { in }(0, T) \\
u_{\varepsilon \mid t=0}=u_{\varepsilon}^{0}, \partial_{t} u_{\varepsilon \mid t=0}=\vartheta_{\varepsilon} \text { in } I \\
u_{\varepsilon} \in L^{\infty}(0, T ; V), \partial_{t} u_{\varepsilon} \in L^{\infty}\left(0, T ; L^{2}(I)\right) .
\end{array}\right.
$$

Our purpose in the present paper is to study the asymptotic behavior of $u_{\varepsilon}$ when $\varepsilon$ tends to zero. First we remark that since for $\varepsilon>0$ small enough, $\rho_{\varepsilon}, a_{\varepsilon}$, are uniformly elliptic and bounded in $W^{1, \infty}\left(0, T ; L^{\infty}(I)\right), B_{\varepsilon}$ is bounded in $L^{\infty}\left(Q_{T}\right)^{2}$, and $u_{\varepsilon}^{0}, \vartheta_{\varepsilon}, f_{\varepsilon}$ bounded in $V, L^{2}(I)$ and $L^{2}\left(Q_{T}\right)$ respectively, we immediately have

Theorem 3.1. For $\varepsilon>0$ small enough, problem (3.1) has a unique solution. Moreover, there exists $C>0$ such that

$$
\left\|u_{\varepsilon}\right\|_{L^{\infty}(0, T ; V)}+\left\|\partial_{t} u_{\varepsilon}\right\|_{L^{\infty}\left(0, T ; L^{2}(I)\right)} \leq C .
$$


Theorem 3.1 implies that, at least for a subsequence, there exists the limit $u_{0}$ of $u_{\varepsilon}$ in the weak-* topology of $L^{\infty}(0, T ; V)$. In order to characterize this limit and to obtain a corrector result for problem (3.1), let us use the two-scale convergence theory.

Definition 3.2. We say that a sequence $v_{\varepsilon} \in L^{2}(I)$ two-scale converges to a function $v \in L^{2}\left(I ; L_{\sharp}^{2}(Y)\right)$ and we write $v_{\varepsilon} \stackrel{2 e}{=} v$, if for every $\psi \in C_{c}\left(I ; C_{\sharp}(Y)\right)$ we have

$$
\lim _{\varepsilon \rightarrow 0} \int_{I} v_{\varepsilon} \psi\left(x, \frac{x}{\varepsilon}\right) \mathrm{d} x=\int_{I} M_{y}(v \psi) \mathrm{d} x .
$$

Analogously, we say that a sequence $v_{\varepsilon} \in L^{2}\left(Q_{T}\right)$ two-scale converges to a function $v \in L^{2}\left(Q_{T} ; L_{\sharp}^{2}(\mathbb{R} \times Y)\right)$ and we write $v_{\varepsilon} \stackrel{2 e}{\rightarrow} v$, if for every $\psi \in C_{c}\left(Q_{T} ; C_{\sharp}(\mathbb{R} \times Y)\right)$ we have

$$
\lim _{\varepsilon \rightarrow 0} \int_{Q_{T}} v_{\varepsilon} \psi\left(t, x, \frac{t}{\varepsilon}, \frac{x}{\varepsilon}\right) \mathrm{d} t \mathrm{~d} x=\int_{Q_{T}} M_{s, y}(v \psi) \mathrm{d} t \mathrm{~d} x .
$$

The interest of the two-scale convergence follows from the classical compactness results which establishes the existence of a two-scale limit for bounded sequences in $L^{2}$ (see $[1,7,8,14-16]$ ). As a consequence of these results and $u_{\varepsilon}^{0}, \vartheta_{\varepsilon}, f_{\varepsilon}$ bounded in $V, L^{2}(I)$ and $L^{2}\left(Q_{T}\right)$, there exist $u^{0} \in V, u^{1} \in L^{2}\left(I ; H_{\sharp}^{1}(Y)\right), \vartheta \in L^{2}\left(I ; L_{\sharp}^{2}(Y)\right)$ and $f \in L^{2}\left(Q_{T} ; L_{\sharp}^{2}(\mathbb{R} \times Y)\right)$ such that, up to a subsequence, we have

$$
u_{\varepsilon}^{0} \rightarrow u^{0} \quad \text { in } V, \quad \partial_{x} u_{\varepsilon}^{0} \stackrel{2 e}{=} \partial_{x} u^{0}+\partial_{y} u^{1}, \quad \vartheta_{\varepsilon} \stackrel{2 e}{\longrightarrow} \vartheta, \quad f_{\varepsilon} \stackrel{2 e}{=} f .
$$

The main result of the paper is given by Theorem 3.4 below which provides the limit function $u_{0}$ of the solution $u_{\varepsilon}$ of (3.1) and the two-scale limit of the sequence $\nabla_{t, x} u_{\varepsilon}$. Related results have been obtained in $[2-6,9,11,12]$. Here the novelty is the analysis of the boundary conditions which (because the waves travel and shock with the walls) influence the behavior of $u_{\varepsilon}$ at the interior of $\Omega$.

Although the problem is periodic, we will prove that the two-scale limit for the whole sequence $\nabla_{t, x} u_{\varepsilon}$ does not exist, instead we will need to consider a subsequence of $\varepsilon$ such that there exist $\alpha^{*}, \beta^{*} \in \mathbb{R} / \mathbb{Z}$ satisfying

$$
\frac{\alpha}{\varepsilon} \rightarrow \alpha^{*}, \quad \frac{\beta}{\varepsilon} \rightarrow \beta^{*} \quad \text { in } \mathbb{R} / \mathbb{Z}
$$

Associated to this subsequence, we define the following spaces which will appear in Theorem 3.4.

Definition 3.3. For a subsequence of $\varepsilon$ such that (3.6) holds, we define the spaces $\mathcal{V}_{1}$ and $\mathcal{V}_{2}$ by

$$
\begin{aligned}
& \mathcal{V}_{1}=H_{0}^{1}\left(0, T ; L^{2}\left(I ; P_{1}\left(\mathcal{W}^{1}\right)\right)\right)
\end{aligned}
$$

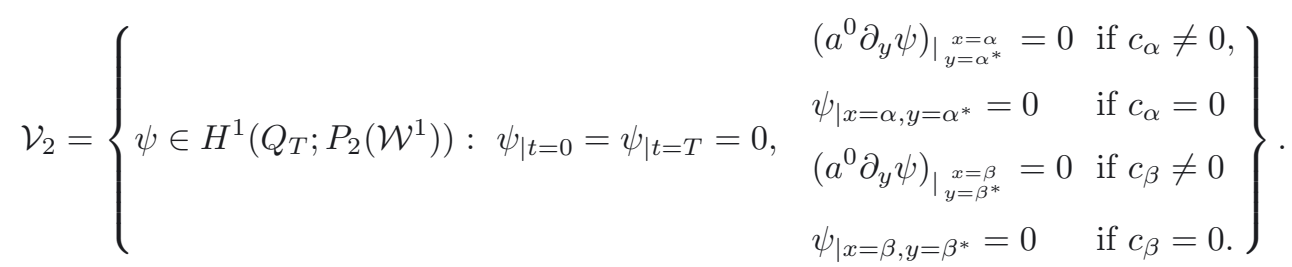

Theorem 3.4. We consider a subsequence of $\varepsilon$, still denoted by $\varepsilon$ such that (3.5) and (3.6) hold. Then, the sequence $u_{\varepsilon}$ satisfies

$$
\begin{gathered}
u_{\varepsilon} \stackrel{*}{\rightarrow} u_{0} \quad \text { in } L^{\infty}(0, T ; V) \\
\nabla_{t, x} u_{\varepsilon} \stackrel{2 e}{\longrightarrow} \nabla_{t, x} u_{0}+\nabla_{s, y} u_{1},
\end{gathered}
$$


where $u_{0}, u_{1}$ are the unique solutions of the variational system

$$
\begin{aligned}
& u_{0} \in L^{\infty}(0, T ; V), \quad \partial_{t} u_{0} \in L^{\infty}\left(0, T ; L^{2}(I)\right) \\
& u_{1} \in L^{\infty}\left(0, T ; L^{2}\left(I ; H_{\sharp}^{1}(\mathbb{R} \times Y)\right)\right), \quad M_{s, y}\left(u_{1}\right)=0 \text { a.e. in } Q_{T} \\
& u_{0 \mid t=0}=u^{0}, \quad\left(\partial_{t} u_{0}+\partial_{s} u_{1}\right)_{\mid t=s=0}=\vartheta, \quad \partial_{y} u_{1 t=s=0}=\partial_{y} u^{1} \\
& \left\{\begin{array}{l}
\int_{Q_{T}} M_{s, y}\left(-\rho^{0}\left(\partial_{t} u_{0} \partial_{t} v_{0}+\partial_{s} u_{1} \partial_{s} v_{1}\right)+a^{0}\left(\partial_{x} u_{0}+\partial_{y} u_{1}\right)\left(\partial_{x} v_{0}+\partial_{y} v_{1}\right)\right) \mathrm{d} t \mathrm{~d} x \\
+\frac{d_{\alpha}}{c_{\alpha}} \int_{\{x=\alpha\}} u_{0} v_{0} \mathrm{~d} t+\frac{d_{\beta}}{c_{\beta}} \int_{\{x=\beta\}} u_{0} v_{0} \mathrm{~d} t \\
+\int_{Q_{T}} M_{s, y}\left(B \cdot\left(\nabla_{t, x} u_{0}+\nabla_{s, y} u_{1}\right) v_{0}\right) \mathrm{d} t \mathrm{~d} x=\int_{Q_{T}} M_{s, y}\left(f v_{0}\right) \mathrm{d} t \mathrm{~d} x \\
\forall v_{0} \in W_{0}^{1,1}\left(0, T ; L^{2}(I)\right) \cap L^{1}(0, T ; V), \quad \forall v_{1} \in L^{2}\left(Q_{T} ; H_{\sharp}^{1}(\mathbb{R} \times Y)\right)
\end{array}\right. \\
& \left\{\begin{array}{l}
\int_{Q_{T}} M_{s, y}\left(-2 \rho^{0} \partial_{s} u_{1} \partial_{t} \psi-\rho^{1}\left(\partial_{t} u_{0}+\partial_{s} u_{1}\right) \partial_{s} \psi+a^{1}\left(\partial_{x} u_{0}+\partial_{y} u_{1}\right) \partial_{y} \psi\right) \mathrm{d} t \mathrm{~d} x \\
+\int_{Q_{T}} M_{s, y}\left(B \cdot\left(\nabla_{t, x} u_{0}+\nabla_{s, y} u_{1}\right) \psi\right) \mathrm{d} t \mathrm{~d} x=\int_{Q_{T}} M_{s, y}(f \psi) \mathrm{d} t \mathrm{~d} x, \quad \forall \psi \in \mathcal{V}_{1}
\end{array}\right. \\
& \left\{\begin{array}{l}
\int_{Q_{T}} M_{s, y}\left(-2 \rho^{0} \partial_{s} u_{1} \partial_{t} \psi+a^{0} \partial_{y} u_{1} \partial_{x} \psi-a^{0} \partial_{x, y}^{2} \psi u_{1}\right) \mathrm{d} t \mathrm{~d} x \\
+\int_{Q_{T}} M_{s, y}\left(-\rho^{1}\left(\partial_{t} u_{0}+\partial_{s} u_{1}\right) \partial_{s} \psi+a^{1}\left(\partial_{x} u_{0}+\partial_{y} u_{1}\right) \partial_{y} \psi\right) \mathrm{d} t \mathrm{~d} x \\
+\int_{Q_{T}} M_{s, y}\left(B \cdot\left(\nabla_{t, x} u_{0}+\nabla_{s, y} u_{1}\right) \psi\right) \mathrm{d} t \mathrm{~d} x=\int_{Q_{T}} M_{s, y}(f \psi) \mathrm{d} t \mathrm{~d} x, \quad \forall \psi \in \mathcal{V}_{2} .
\end{array}\right.
\end{aligned}
$$

Remark 3.5. In the case where $c_{\alpha}=0$, the term

$$
\frac{d_{\alpha}}{c_{\alpha}} \int_{\{x=\alpha\}} u_{0} v_{0} \mathrm{~d} t
$$

which appears in (3.14) must be understood as zero. Observe that in this case, the functions $u_{0}$ and $v_{0}$ vanish at $x=\alpha$. Analogously, if $c_{\beta}=0$, the term

$$
\frac{d_{\beta}}{c_{\beta}} \int_{\{x=\beta\}} u_{0} v_{0} \mathrm{~d} t
$$

is defined as zero.

Remark 3.6. In order to have uniqueness for $u_{1}$ we have taken it satisfying $M_{s, y}\left(u_{1}\right)=0$. Clearly, (3.10) still holds adding to $u_{1}$ any function independent of the variables $(s, y)$.

Remark 3.7. Observe that the two-scale limit of the solution $u_{\varepsilon}$ of (3.1) depends on the position of the extremes of $I$ with respect to the periodic cell, which correspond to the points $\alpha^{*}$ and $\beta^{*}$ given by (3.6). 
Remark 3.8. In equations (3.15) and (3.16) the function $\psi$ applies $Q_{T}$ into the spaces $P_{1}\left(\mathcal{W}^{1}\right)$ and $P_{2}\left(\mathcal{W}^{1}\right)$ respectively. This shows that the behavior of elementary waves associated to simple or multiple eigenvalues of (2.4) is different.

Remark 3.9. From (3.14), with $v_{0}=0$, we deduce the equation

$$
\rho^{0} \partial_{s s}^{2} u_{1}-\partial_{y}\left(a^{0}\left(\partial_{x} u_{0}+\partial_{y} u_{1}\right)\right)=0 \text { in } \mathbb{R} \times \mathbb{R}, \text { a.e. in } Q_{T} .
$$

A solution of this problem independent of $s$ is given by the elliptic corrector $\hat{u}_{1}$ (see e.g. $\left.[1,4,14]\right)$ solution of

$$
\left\{\begin{array}{l}
\hat{u}_{1} \in L^{\infty}\left(0, T ; L^{2}\left(I ; H_{\sharp}^{1}(Y)\right)\right), \quad M_{y}\left(\hat{u}_{1}\right)=0 \text { a.e. }(t, x) \in Q_{T} \\
-\partial_{y}\left(a^{0}\left(\partial_{x} u_{0}+\partial_{y} \hat{u}_{1}\right)\right)=0 \text { in } \mathbb{R}, \quad \text { a.e. in } Q_{T},
\end{array}\right.
$$

or equivalently

$$
\partial_{y} \hat{u}_{1}(t, x, y)=a_{h}^{0}\left(\frac{1}{a^{0}(y)}-\frac{1}{a_{h}^{0}}\right) \partial_{x} u_{0}(t, x), \quad \text { a.e. }(t, x, y) \in Q_{T}, \times \mathbb{R}, \quad M_{y}\left(\hat{u}_{1}\right)=0,
$$

with $a_{h}^{0}$ the homogenized coefficient corresponding to $a^{0}$, i.e.

$$
a_{h}^{0}=\frac{1}{M_{y}\left(\frac{1}{a^{0}}\right)} .
$$

From (3.17) and (3.18), we deduce that $u_{1}$ can be decomposed as

$$
u_{1}=\hat{u}_{1}+\tilde{u}_{1},
$$

with

$$
\tilde{u}_{1} \in L^{\infty}\left(0, T ; L^{2}\left(I ; \mathcal{W}^{1}\right)\right) .
$$

Remark 3.10. Taking $v_{1}=0$ in (3.14) and using (3.21), (3.19) and $M_{s}\left(\tilde{u}_{1}\right)=0$, which is a consequence of (3.22), we deduce that $u_{0}$ satisfies

$$
\left\{\begin{array}{l}
M_{y}\left(\rho^{0}\right) \partial_{t t}^{2} u_{0}-a_{h}^{0} \partial_{x x}^{2} u_{0}+B_{h} \cdot \nabla_{t, x} u_{0}+M_{s, y}\left(B \cdot \nabla_{s, y} \tilde{u}_{1}\right)=M_{s, y}(f) \text { in } Q_{T} \\
\left(-c_{\alpha} a_{h}^{0} \partial_{x} u_{0}+d_{\alpha} u_{0}\right)_{\mid x=\alpha}=0,\left(c_{\beta} a_{h}^{0} \partial_{x} u_{0}+d_{\beta} u_{0}\right)_{\mid x=\beta}=0 \quad \text { in }(0, T) \\
u_{0 \mid t=0}=u^{0}
\end{array}\right.
$$

where, denoting by $b_{t}, b_{x}$ the two components of $B$, the function $B_{h}$ is given by

$$
B_{h}=\left(b_{h, t}, b_{h, x}\right) \quad \text { with } b_{h, t}=M_{s, y}\left(b_{t}\right), \quad b_{h, x}=\frac{M_{s, y}\left(\frac{b_{x}}{a^{0}}\right)}{M_{y}\left(\frac{1}{a^{0}}\right)} .
$$

If we assume that $B$ satisfies

$$
M_{s, y}\left(B \cdot \nabla_{s, y} v\right)=0, \quad \forall v \in \mathcal{W}^{1}, \text { a.e. in } Q_{T},
$$

then the fourth term in the PDE in (3.23) vanishes providing an equation for $u_{0}$ (the limit equation). This holds, for example, if $B$ does not depend on $s$. In general (3.25) is not satisfied and then, since $\tilde{u}_{1}$ does not depend locally on $u_{0}$, we get a non-local equation for $u_{0}$. An example of this situation is given in [10], where $Q_{T}=(0, T) \times \mathbb{R}^{N}$. 
Remark 3.11. Equation (3.23) for $u_{0}$ must be completed with initial conditions for $u_{0}$ and $\partial_{t} u_{0}$. The first one is contained in (3.13). The second one can be obtained as follows: Using (3.21), we can write the second equation in (3.13) as

$$
\left(\partial_{t} u_{0}+\partial_{s} \tilde{u}_{1}\right)_{\mid t=s=0}=\vartheta
$$

where $\partial_{s} \tilde{u}_{1}$ is a combination of eigenfunctions of problem (2.7) corresponding to non-vanishing eigenvalues. Thus $M_{y}\left(\rho^{0} \partial_{s} \tilde{u}_{1}\right)=0$ and therefore, multiplying (3.26) by $\rho^{0}$ and taking the mean value in $y$ we get

$$
\partial_{t} u_{0 \mid t=0}=\frac{M_{y}\left(\rho^{0} \vartheta\right)}{M_{y}\left(\rho^{0}\right)}
$$

Equation (3.16) implicitly provides boundary conditions for the function $\tilde{u}_{1}$ given by (3.21). This is given by the following Proposition which shows that $P_{2} \tilde{u}_{1}$ satisfies the boundary conditions imposed to $\psi$ in (3.16).

Proposition 3.12. In the conditions of Theorem 3.4, and defining $\tilde{u}_{1}$ by (3.21), we have

$$
\begin{aligned}
& \left(a^{0} \partial_{y} P_{2} \tilde{u}_{1}\right)_{\mid x=\alpha, y=\alpha^{*}}=0 \quad \text { if } c_{\alpha} \neq 0, \quad P_{2} \tilde{u}_{1 \mid x=\alpha, y=\alpha^{*}}=0 \quad \text { if } c_{\alpha}=0, \\
& \left(a^{0} \partial_{y} P_{2} \tilde{u}_{1}\right)_{\mid x=\beta, y=\beta^{*}}=0 \quad \text { if } c_{\beta} \neq 0, \quad P_{2} \tilde{u}_{1 \mid x=\beta, y=\beta^{*}}=0 \quad \text { if } c_{\beta}=0 .
\end{aligned}
$$

From Theorem 3.4 it is also possible to obtain a corrector result for problem (3.1). For this purpose, it is necessary to assume that in (3.5) the two-scale convergence holds in the following strong sense:

$$
\left\{\begin{array}{l}
\lim _{\varepsilon \rightarrow 0} \int_{I} \partial_{x} u_{\varepsilon}^{0} \psi_{\varepsilon} \mathrm{d} x=\int_{I} M_{y}\left(\left(\partial_{x} u^{0}+\partial_{y} u^{1}\right) \psi\right) \mathrm{d} x \\
\lim _{\varepsilon \rightarrow 0} \int_{I} \vartheta_{\varepsilon} \psi_{\varepsilon} \mathrm{d} x=\int_{I} M_{y}(\vartheta \psi) \mathrm{d} x \\
\forall \psi_{\varepsilon} \text { bounded in } L^{2}(I)^{N}, \psi \in L^{2}\left(I ; L_{\sharp}^{2}(Y)\right), \quad \Psi_{\varepsilon} \stackrel{2 e}{=} \psi,
\end{array}\right.
$$

which is possible to prove (see e.g. [1]) that it is equivalent to assume that the second and third assertions in (3.5) hold and

$$
\lim _{\varepsilon \rightarrow 0} \int_{I}\left|\partial_{x} u_{\varepsilon}^{0}\right|^{2} \mathrm{~d} x=\int_{I} M_{y}\left(\left|\partial_{x} u^{0}\right|^{2}+\left|\partial_{y} u^{1}\right|^{2}\right) \mathrm{d} x, \quad \lim _{\varepsilon \rightarrow 0} \int_{I}\left|\vartheta_{\varepsilon}\right|^{2} \mathrm{~d} x=\int_{I} M_{y}\left(|\vartheta|^{2}\right) \mathrm{d} x .
$$

The corrector result is given by Theorem 3.13 below. Its proof is very similar to the one of Theorem 3.7 in [10] and then it will not be given here. The main idea consists in using Theorem 3.4 to pass to the limit in the energy identity for problem (3.1).

Theorem 3.13. We assume that in Theorem 3.4, the sequences $u_{\varepsilon}^{0}$ and $\vartheta_{\varepsilon}$ satisfy (3.29), then, for every sequence $\Gamma_{\varepsilon} \in L^{2}\left(Q_{T}\right)^{2}$ which two-scale converges strongly to $\nabla_{s, y} u_{1}$, we have

$$
\lim _{\varepsilon \rightarrow 0} \int_{Q_{T}}\left|\nabla_{t, x}\left(u_{\varepsilon}-u_{0}\right)-\Gamma_{\varepsilon}\right|^{2} \mathrm{~d} x \mathrm{~d} t=0 .
$$

Remark 3.14. If the function $\nabla_{s, y} u_{1}$ is in $L^{2}\left(Q_{T} ; C_{\sharp}^{0}(\mathbb{R} \times Y)^{2}\right)$, then we can take in (3.30)

$$
\Gamma_{\varepsilon}=\nabla_{s, y} u_{1}\left(t, x, \frac{t}{\varepsilon}, \frac{x}{\varepsilon}\right) .
$$

In this case Theorem 3.13 asserts

$$
\nabla_{t, x} u_{\varepsilon}-\nabla_{t, x} u_{0}-\nabla_{s, y} u_{1}\left(t, x, \frac{t}{\varepsilon}, \frac{x}{\varepsilon}\right) \rightarrow 0 \quad \text { in } L^{2}\left(Q_{T}\right)^{2},
$$


which assuming further regularity in $u_{1}$ reads as

$$
u_{\varepsilon}-u_{0}-\varepsilon u_{1}\left(t, x, \frac{t}{\varepsilon}, \frac{x}{\varepsilon}\right) \rightarrow 0 \text { in } H^{1}\left(Q_{T}\right)
$$

We finish this section with a simplified model of (3.1) where we can explicitly obtain the function $u_{1}$ which appears in Theorem 3.4 from $u_{0}$, the initial data and the right-hand side. For this purpose, let us consider an orthonormal basis of the spaces $W_{j}$ composed by real functions. Namely:

If $j \in J_{1}$, we consider a real eigenfunction $\Phi_{j} \in W_{j}$ such that

$$
M_{y}\left(\rho^{0}\left|\Phi_{j}\right|^{2}\right)=1 .
$$

If $j \in J_{2}$, we consider two real eigenfunctions $\Phi_{j 1}, \Phi_{j 2} \in W_{j}$ such that

$$
M_{y}\left(\rho^{0} \Phi_{j k} \Phi_{j l}\right)=\delta_{k l}, \quad k, l \in\{1,2\} .
$$

Since $W_{j}=W_{-j}$ for every $j \in \mathbb{Z} \backslash\{0\}$, we can also assume

$$
\Phi_{j}=\Phi_{-j}, \forall j \in J_{1}, \quad \Phi_{j 1}=\Phi_{-j 1}, \Phi_{j 2}=\Phi_{-j 2}, \forall j \in J_{2} .
$$

Moreover, we remark that these functions $\Phi_{j}, \Phi_{j 1}, \Phi_{j 2}$ with $j \in \mathbb{Z}^{+}$are a basis of $L_{\sharp}^{2}(Y) / \mathbb{R}$ and $H_{\sharp}^{1}(Y) / \mathbb{R}$.

Taking into account that $f$ belongs to $L^{2}\left(Q_{T} ; C_{\sharp}(\mathbb{R} \times Y)\right)$, and then to the space $L^{2}\left(Q_{T} ; L_{\sharp}^{2}(\mathbb{R} \times Y)\right)$, that $u^{1}$ belongs to $L^{2}\left(I ; H_{\sharp}^{1}(Y)\right)$, and can be chosen with zero mean value in $y$, and that $\vartheta$ belongs to $L^{2}\left(I ; L_{\sharp}^{2}(Y)\right)$, we can decompose these functions as

$$
\begin{aligned}
& \left\{\begin{aligned}
\frac{f(t, x, s, y)}{\rho^{0}(y)}= & \sum_{j \in J_{1}} f_{j}(t, x) \Phi_{j}(y) \mathrm{e}^{\mathrm{i} \lambda_{j} s} \\
& +\sum_{j \in J_{2}}\left(f_{j 1}(t, x) \Phi_{j 1}(y)+f_{j 2}(t, x) \Phi_{j 2}(y)\right) \mathrm{e}^{\mathrm{i} \lambda_{j} s}+f^{*}(t, x, s, y)
\end{aligned}\right. \\
& u^{1}(x, y)=\sum_{j \in J_{1}^{+}} u_{j}^{1}(x) \Phi_{j}(y)+\sum_{j \in J_{2}^{+}}\left(u_{j 1}^{1}(x) \Phi_{j 1}(y)+u_{j 2}^{1}(x) \Phi_{j 2}(y)\right) \\
& \vartheta(x, y)=\sum_{j \in J_{1}^{+}} \vartheta_{j}(x) \Phi_{j}(y)+\sum_{j \in J_{2}^{+}}\left(\vartheta_{j 1}(x) \Phi_{j 1}(y)+\vartheta_{j 2}(x) \Phi_{j 2}(y)\right)+M_{y}(\vartheta),
\end{aligned}
$$

with

$$
\begin{gathered}
M_{s, y}\left(\rho^{0} f^{*} v\right)=0 \text { a.e. in } Q_{T}, \quad \forall v \in \mathcal{W}^{0} \\
\sum_{j \in J_{1}}\left\|f_{j}\right\|_{L^{2}\left(Q_{T}\right)}^{2}+\sum_{j \in J_{2}}\left(\left\|f_{j 1}\right\|_{L^{2}\left(Q_{T}\right)}^{2}+\left\|f_{j^{2}}\right\|_{L^{2}\left(Q_{T}\right)}^{2}\right)<+\infty \\
\sum_{j \in J_{1}^{+}} \lambda_{j}^{2}\left\|u_{j}^{1}\right\|_{L^{2}(I)}^{2}+\sum_{j \in J_{2}^{+}} \lambda_{j}^{2}\left(\left\|u_{j_{1}}^{1}\right\|_{L^{2}(I)}^{2}+\left\|u_{j 2}^{1}\right\|_{L^{2}(I)}^{2}\right)<+\infty \\
\sum_{j \in J_{1}^{+}}\left\|\vartheta_{j}\right\|_{L^{2}(I)}^{2}+\sum_{j \in J_{2}^{+}}\left(\left\|\vartheta_{j 1}\right\|_{L^{2}(I)}^{2}+\left\|\vartheta_{j 2}\right\|_{L^{2}(I)}^{2}\right)<+\infty .
\end{gathered}
$$


We also denote (recall that by (2.8) the definition of $\kappa_{j}$ below does not depend on $y$ )

$$
\begin{gathered}
m_{j}=M_{y}\left(a^{0} \partial_{y} \Phi_{j}\right), \quad j \in J_{1}, \quad m_{j l}=M_{y}\left(a^{0} \partial_{y} \Phi_{j l}\right), \quad j \in J_{2}, l \in\{1,2\} \\
\kappa_{j}=a^{0}(y)\left(\Phi_{j 1}(y) \frac{\mathrm{d} \Phi_{j 2}}{\mathrm{~d} y}(y)-\Phi_{j 2}(y) \frac{\mathrm{d} \Phi_{j 1}}{\mathrm{~d} y}(y)\right), \quad \text { a.e. } y \in \mathbb{R} \\
\mu_{j l}^{\alpha^{*}}=\Phi_{j l}\left(\alpha^{*}\right), \quad \mu_{j l}^{\beta^{*}}=\Phi_{j l}\left(\beta^{*}\right), \quad j \in J_{2}, l \in\{1,2\} \\
\nu_{j l}^{\alpha^{*}}=\left(a_{0} \frac{\mathrm{d} \Phi_{j l}}{\mathrm{~d} y}\right)\left(\alpha^{*}\right), \quad \nu_{j l}^{\beta^{*}}=\left(a_{0} \frac{\mathrm{d} \Phi_{j l}}{\mathrm{~d} y}\right)\left(\beta^{*}\right), \quad j \in J_{2}, l \in\{1,2\} .
\end{gathered}
$$

Theorem 3.15. We assume that the functions $\rho^{1}, a^{1}, B$ in Theorem 3.4 vanish. Then the function $u_{1}$ is given by (3.21), where the functions $\hat{u}_{1}$ and $\tilde{u}_{1}$ can be written as

$$
\begin{gathered}
\hat{u}_{1}(t, x, y)=\sum_{j \in J_{1}^{+}} \hat{\mathrm{u}}_{j}(t, x) \Phi_{j}(y)+\sum_{j \in J_{2}^{+}}\left(\hat{\mathrm{u}}_{j 1}(t, x) \Phi_{j 1}(y)+\hat{\mathrm{u}}_{j 2}(t, x) \Phi_{j 2}(y)\right) \\
\tilde{u}_{1}(t, x, s, y)=\sum_{j \in J_{1}} \tilde{\mathrm{u}}_{j}(t, x) \Phi_{j}(y) \mathrm{e}^{\mathrm{i} \lambda_{j} s}+\sum_{j \in J_{2}}\left(\tilde{\mathrm{u}}_{j 1}(t, x) \Phi_{j 1}(y)+\tilde{\mathrm{u}}_{j 2}(t, x) \Phi_{j 2}(y)\right) \mathrm{e}^{\mathrm{i} \lambda_{j} s},
\end{gathered}
$$

with

$$
\begin{aligned}
& \operatorname{supess}_{t \in(0, T)}\left(\sum_{j \in J_{1}^{+}} \lambda_{j}^{2}\left\|\hat{\mathrm{u}}_{j}(t, x)\right\|_{L^{2}(I)}^{2}+\sum_{j \in J_{2}^{+}} \lambda_{j}^{2}\left(\left\|\hat{\mathrm{u}}_{j 1}(t, x)\right\|_{L^{2}(I)}^{2}+\left\|\hat{\mathrm{u}}_{j 2}(t, x)\right\|_{L^{2}(I)}^{2}\right)\right)<\infty \\
& \operatorname{supess}_{t \in(0, T)}\left(\sum_{j \in J_{1}} \lambda_{j}^{2}\left\|\tilde{\mathbf{u}}_{j}(t, x)\right\|_{L^{2}(I)}^{2}+\sum_{j \in J_{2}} \lambda_{j}^{2}\left(\left\|\tilde{\mathbf{u}}_{j 1}(t, x)\right\|_{L^{2}(I)}^{2}+\left\|\tilde{\mathbf{u}}_{j 2}(t, x)\right\|_{L^{2}(I)}^{2}\right)\right)<\infty .
\end{aligned}
$$

The coefficients $\hat{\mathrm{u}}_{j}$ and $\hat{\mathrm{u}}_{j l}$ are given by

$$
\begin{gathered}
m_{j} \partial_{x} u_{0}+\lambda_{j}^{2} \hat{\mathrm{u}}_{j}=0, \text { a.e. in } I, \forall t \in[0, T], \forall j \in J_{1}^{+} \\
m_{j l} \partial_{x} u_{0}+\lambda_{j}^{2} \hat{\mathrm{u}}_{j l}=0, \text { a.e. in } I, \forall t \in[0, T], \forall j \in J_{2}^{+}, \forall l \in\{1,2\} .
\end{gathered}
$$

For $j \in J_{1}^{+}$, the coefficients $\tilde{\mathbf{u}}_{j}, \tilde{\mathrm{u}}_{-j}$ are given by

$$
\begin{gathered}
\tilde{\mathrm{u}}_{j}(t, x)=\frac{1}{2}\left(u_{j}^{1}(x)+\frac{m_{j}}{\lambda_{j}^{2}} \partial_{x} u_{0}(0, x)-\frac{\vartheta_{j}(x)}{\lambda_{j}} i\right)-\frac{i}{2 \lambda_{j}} \int_{0}^{t} f_{j}(\tau, x) d \tau, \text { a.e. in } Q_{T} \\
\tilde{\mathrm{u}}_{-j}(t, x)=\frac{1}{2}\left(u_{j}^{1}(x)+\frac{m_{j}}{\lambda_{j}^{2}} \partial_{x} u_{0}(0, x)+\frac{\vartheta_{j}(x)}{\lambda_{j}} i\right)-\frac{i}{2 \lambda_{j}} \int_{0}^{t} f_{j}(\tau, x) d \tau, \text { a.e. in } Q_{T} .
\end{gathered}
$$

The coefficients $\tilde{\mathrm{u}}_{j l}$ are the solutions of the system

$$
\left\{\begin{array}{l}
2 i \lambda_{j} \partial_{t} \tilde{\mathbf{u}}_{j 1}-\kappa_{j} \partial_{x} \tilde{\mathbf{u}}_{j 2}=f_{j 1} \\
2 i \lambda_{j} \partial_{t} \tilde{\mathbf{u}}_{j 2}+\kappa_{j} \partial_{x} \tilde{\mathbf{u}}_{j 1}=f_{j 2},
\end{array}\right.
$$


with the boundary conditions

$$
\left\{\begin{array}{l}
\left(\nu_{j 1}^{\alpha^{*}} \tilde{\mathrm{u}}_{j 1}+\nu_{j 2}^{\alpha^{*}} \tilde{\mathrm{u}}_{j 2}\right)_{\mid x=\alpha}=0 \text { if } c_{\alpha} \neq 0, \quad\left(\mu_{j 1}^{\alpha^{*}} \tilde{\mathrm{u}}_{j 1}+\mu_{j 2}^{\alpha^{*}} \tilde{\mathrm{u}}_{j 2}\right)_{\mid x=\alpha}=0 \text { if } c_{\alpha}=0 \\
\left(\nu_{j 1}^{\beta^{*}} \tilde{\mathrm{u}}_{j 1}+\nu_{j 2}^{\beta^{*}} \tilde{\mathrm{u}}_{j 2}\right)_{\mid x=\beta}=0 \text { if } c_{\beta} \neq 0, \quad\left(\mu_{j 1}^{\beta^{*}} \tilde{\mathrm{u}}_{j 1}+\mu_{j 2}^{\beta^{*}} \tilde{\mathrm{u}}_{j 2}\right)_{\mid x=\beta}=0 \text { if } c_{\beta}=0,
\end{array}\right.
$$

and the initial conditions

$$
\begin{gathered}
\tilde{\mathbf{u}}_{j l \mid t=0}=\frac{1}{2}\left(u_{j l}^{1}+\frac{m_{j l}}{\lambda_{j}^{2}} \partial_{x} u_{0 \mid t=0}-\frac{\vartheta_{j l}}{\lambda_{j}} i\right), \text { a.e. in } I, \forall j \in J_{2}^{+}, l=1,2 \\
\tilde{\mathbf{u}}_{-j l \mid t=0}=\frac{1}{2}\left(u_{j l}^{1}+\frac{m_{j l}}{\lambda_{j}^{2}} \partial_{x} u_{0 \mid t=0}+\frac{\vartheta_{j l}}{\lambda_{j}} i\right), \text { a.e. in } I, \forall j \in J_{2}^{+}, l=1,2 .
\end{gathered}
$$

Remark 3.16. The existence and uniqueness of solution for system (3.53) with the boundary conditions given by (3.54) and the initial conditions given by (3.55) and (3.56), follows from Theorem 3.4. It can also be proved directly. For this purpose we remark that it is easy to obtain an a priori estimate for this problem. Namely, assume to simplify $c_{\alpha}=c_{\beta}=0$ (Dirichlet conditions), the other cases are similar. Then, multiplying the first equation in (3.53) by $\overline{\tilde{u}}_{j 1}$, the second equation by $\overline{\tilde{u}}_{j 2}$, integrating in $(\alpha, \beta)$, dividing by $2 i \lambda_{j}$ and adding the two equations, we get

$$
\int_{\alpha}^{\beta}\left(\partial_{t} \tilde{\mathbf{u}}_{j 1} \overline{\tilde{u}}_{j 1}+\partial_{t} \tilde{\mathbf{u}}_{j 2} \overline{\tilde{u}}_{j 2}\right) \mathrm{d} x+\frac{\kappa_{j} i}{2 \lambda_{j}} \int_{\alpha}^{\beta}\left(\partial_{x} \tilde{\mathbf{u}}_{j 2} \overline{\tilde{\mathbf{u}}}_{j 1}-\partial_{x} \tilde{\mathbf{u}}_{j 1} \overline{\tilde{u}}_{j 2}\right) \mathrm{d} x=-\frac{i}{2 \lambda_{j}} \int_{\alpha}^{\beta}\left(f_{j 1} \overline{\tilde{u}}_{j 1}+f_{j 2} \overline{\tilde{u}}_{j 2}\right) \mathrm{d} x .
$$

In the second term an integration by parts shows

$$
\int_{\alpha}^{\beta}\left(\partial_{x} \tilde{\mathbf{u}}_{j 2} \overline{\tilde{\mathbf{u}}}_{j 1}-\partial_{x} \tilde{\mathbf{u}}_{j 1} \overline{\tilde{\mathbf{u}}}_{j 2}\right) \mathrm{d} x=2 \int_{\alpha}^{\beta} \mathcal{R} e\left(\partial_{x} \tilde{\mathbf{u}}_{j 2} \overline{\tilde{\mathbf{u}}}_{j 1}\right) \mathrm{d} x-\tilde{\mathbf{u}}_{j 1}(t, \beta) \overline{\tilde{\mathbf{u}}}_{j 2}(t, \beta)+\tilde{\mathbf{u}}_{j 1}(t, \alpha) \overline{\tilde{u}}_{j 2}(t, \alpha) .
$$

Now, we observe that if $\mu_{j 1}^{\alpha^{*}}=0$ then $\tilde{\mathrm{u}}_{j 2}(t, \alpha)=0$ by (3.54) and therefore

$$
\tilde{\mathrm{u}}_{j 1}(t, \alpha) \overline{\tilde{\mathbf{u}}}_{j 2}(t, \alpha)=0 \in \mathbb{R} .
$$

If $\mu_{j 1}^{\alpha^{*}} \neq 0$, then, multiplying the first equation in (3.54) by $\overline{\tilde{u}}_{j 2}(t, \alpha)$ we have

$$
\tilde{\mathrm{u}}_{j 1}(t, \alpha) \overline{\tilde{u}}_{j 2}(t, \alpha)=-\frac{\mu_{j 2}^{\alpha^{*}}}{\mu_{j 1}^{\alpha^{*}}}\left|\tilde{\mathrm{u}}_{j 2}(t, \alpha)\right|^{2} \in \mathbb{R} .
$$

So, we always have that the third term on the right-hand side of (3.58) is real. Analogously, we can also show that the second term is real and therefore (3.58) shows that the second term in (3.57) is purely imaginary. Thus, taking the real part in (3.57) we get

$$
\frac{1}{2} \frac{\mathrm{d}}{\mathrm{d} t} \int_{\alpha}^{\beta}\left(\left|\tilde{\mathrm{u}}_{j 1}\right|^{2}+\left|\tilde{\mathrm{u}}_{j 2}\right|^{2}\right) \mathrm{d} x \leq \frac{1}{2 \lambda_{j}} \int_{\alpha}^{\beta}\left(\left|f_{j 1}\right|^{2}+\left|f_{j 2}\right|^{2}\right)^{\frac{1}{2}}\left(\left|\tilde{\mathrm{u}}_{j 1}\right|^{2}+\left|\tilde{\mathrm{u}}_{j 2}\right|^{2}\right)^{\frac{1}{2}} \mathrm{~d} x
$$

This estimate allows us to use Gronwall's inequality to deduce the a priori estimate

$$
\sum_{k=1}^{2}\left\|\tilde{\mathrm{u}}_{j k}\right\|_{L^{\infty}\left(0, T ; L^{2}(\alpha, \beta)\right.} \leq \frac{C}{\lambda_{j}} \sum_{k=1}^{2}\left\|f_{j k}\right\|_{L^{2}\left(Q_{T}\right)}+C\left\|u_{j}^{1}\right\|_{L^{2}(\alpha, \beta)}+\frac{C}{\lambda_{j}}\left\|\theta_{j}^{1}\right\|_{L^{2}(\alpha, \beta)}+\frac{C}{\lambda_{j}^{2}}\left\|\partial_{x} u_{0 \mid t=0}\right\|_{L^{2}(\alpha, \beta)} .
$$

Then, thanks to this a priori estimate and using for example a Galerkin approximation, is now easy to prove existence and uniqueness of solution for (3.53)-(3.56). 
Remark 3.17. From (3.53), we can deduce that the functions $\tilde{u}_{j 1}, \tilde{u}_{j 2}$ are the solutions of the wave equations

$$
\begin{gathered}
4 \lambda_{j}^{2} \partial_{t t}^{2} \tilde{\mathbf{u}}_{j 1}-\kappa_{j}^{2} \partial_{x x}^{2} \tilde{\mathbf{u}}_{j 1}=-2 i \lambda_{j} \partial_{t} f_{j 1}-\kappa_{j} \partial_{x} f_{j 2} \\
4 \lambda_{j}^{2} \partial_{t t}^{2} \tilde{\mathbf{u}}_{j 2}-\kappa_{j}^{2} \partial_{x x}^{2} \tilde{\mathbf{u}}_{j 2}=\kappa_{j} \partial_{x} f_{j 1}-2 i \lambda_{j} \partial_{t} f_{j 2},
\end{gathered}
$$

combined with some boundary and initial conditions which can also be deduced from (3.54) and (3.55), (3.56). The right-hand side of (3.59) and (3.60) has a lack of smoothness (something similar happens for the initial and the boundary conditions), the corresponding solution can be defined by transposition.

Remark 3.18. In Theorem 3.15 we can observe how the physical behavior of the elementary waves corresponding to frequencies $\lambda_{j}$ is completely different in the case of simple or multiple eigenvalues. Namely, if $\lambda_{j}$ belongs to $J_{1}$ the corresponding coefficient $\tilde{u}_{j}$ of $\tilde{u}$ satisfies the equation (see 3.51 )

$$
2 \lambda_{j} i \partial_{t} \tilde{u}_{j}=f_{j} .
$$

It corresponds to a perturbation which does not moves in space, such as it can be observed in (3.51) which provides a value for $\tilde{u}_{j}$ in a point $(t, x)$ depending only on the values of the data for the same spatial point $x$. Thus, the behavior of these wave does not depend on the boundary conditions.

When $\lambda_{j}$ is an eigenvalue of multiplicity two, the equations corresponding to the coefficients $\tilde{u}_{j 1}, \tilde{u}_{j 2}$ are given by (3.59) and (3.60). They correspond to waves moving in the space at a velocity $\kappa_{j} /\left(2 \lambda_{j}\right)$ through the characteristic

$$
x= \pm \frac{\kappa_{j}}{2 \lambda_{j}} t .
$$

Thus, perturbations originated in a certain point of $(\alpha, \beta)$ arrive to the boundary in a finite time and there, they are reflected depending on the chosen boundary conditions.

\section{Proof of the Results}

This section is devoted to show the different results stated in the previous one.

In order to prove Theorem 3.4, we need the following lemma which is a particular case of Lemma 4.1 in [10] and then it is given without proof.

Lemma 4.1. We consider a bounded sequence $\zeta_{\varepsilon}$ in $H^{1}\left(0, T ; L^{2}(I)\right)$ such that there exist $\zeta_{0} \in$ $H^{1}\left(0, T ; L^{2}\left(I ; L_{\sharp}^{2}(Y)\right)\right.$ and $\zeta_{1} \in L^{2}\left(0, T ; L^{2}\left(I ; H_{\sharp}^{1}\left(\mathbb{R} ; L_{\sharp}^{2}(Y)\right)\right)\right.$ satisfying

$$
\zeta_{\varepsilon} \stackrel{2 e}{=} \zeta_{0}, \quad \partial_{t} \zeta_{\varepsilon} \stackrel{2 e}{=} \partial_{t} \zeta_{0}+\partial_{s} \zeta_{1}
$$

Then,

$$
\zeta_{\varepsilon}(t, .) \stackrel{2 e}{\longrightarrow} \zeta_{0}(t, ., .), \quad \forall t \in[0, T] .
$$

Besides, for every $\varphi \in C^{1}\left(\overline{Q_{T}}\right), \varphi_{\mid t=0}=\varphi_{\mid t=T}=0, h \in L_{\sharp}^{2}(Y)$ and $g \in W^{1, \infty}(\mathbb{R})$, periodic with period $S$, for some $S>0$, and zero mean value, we have

$$
\exists \lim _{\varepsilon \rightarrow 0} \frac{1}{\varepsilon} \int_{Q_{T}} \zeta_{\varepsilon}(t, x) \varphi(t, x) g\left(\frac{t}{\varepsilon}\right) h\left(\frac{x}{\varepsilon}\right) \mathrm{d} t \mathrm{~d} x=\int_{Q_{T}} M_{s, y}\left(\zeta_{1}(t, x, s, y) \varphi(t, x) g(s) h(y)\right) \mathrm{d} t \mathrm{~d} x .
$$

Proof of Theorem 3.4. We split the proof in several steps.

Step 1. From (3.2), we know that, at least for a subsequence, there exists $u_{0} \in L^{\infty}(0, T ; V)$, with $\partial_{t} u_{0} \in$ $L^{\infty}\left(0, T ; L^{2}(I)\right)$ such that

$$
u_{\varepsilon} \stackrel{*}{\rightarrow} u_{0} \quad \text { in } L^{\infty}(0, T ; V), \quad \partial_{t} u_{\varepsilon} \stackrel{*}{\rightarrow} \partial_{t} u_{0} \quad \text { in } L^{\infty}\left(0, T ; L^{2}(I)\right) .
$$


Moreover, the compactness property of the two-scale convergence (see $[1,7,8,14-16])$ implies the existence of $u_{1} \in L^{\infty}\left(0, T ; L^{2}\left(I ; H_{\sharp}^{1}(\mathbb{R} \times Y)\right)\right), u_{1}=u_{1}(t, x, s, y)$, such that

$$
\nabla_{t, x} u_{\varepsilon} \stackrel{2 \mathrm{e}}{=} \nabla_{t, x} u_{0}+\nabla_{s, y} u_{1}
$$

The problem is to characterize these functions. For this purpose, as it is usual in the two-scale convergence method we take as test function in (3.1) the sequence

$$
v_{\varepsilon}(t, x)=v_{0}(t, x)+\varepsilon v_{1}\left(t, x, \frac{t}{\varepsilon}, \frac{x}{\varepsilon}\right)
$$

with $v_{0} \in C_{c}^{\infty}(0, T ; V), v_{1} \in C_{c}^{\infty}\left(Q_{T} ; C_{\sharp}^{\infty}(\mathbb{R} \times Y)\right)$. Passing to the limit when $\varepsilon$ tends to zero we easily deduce that $u_{0}, u_{1}$ satisfy (3.14). However this is not enough to characterize the function $u_{1}$ and then we need to use another type of test functions. For this purpose, we reason as in [10]. Namely, for $j \in \mathbb{Z} \backslash\{0\}, \Phi \in W_{j}$ and $\varphi \in C^{\infty}\left([0, T] ; C_{c}^{\infty}(I)\right), \varphi_{\mid t=T}=0$ we take as test function in (3.1) the sequence

$$
v_{\varepsilon}(t, x)=\varphi(t, x) \mathrm{e}^{\mathrm{i} \lambda_{j} \frac{t}{\varepsilon}} \Phi\left(\frac{x}{\varepsilon}\right), \text { a.e. }(t, x) \in Q_{T} .
$$

Then, the same calculus which appears in Step 3 of the proof of Theorem 3.5 in [10] shows

$$
\begin{aligned}
& -\int_{I} M_{y}(\vartheta \varphi(0, x) \Phi) \mathrm{d} x-2 \int_{Q_{T}} M_{s, y}\left(\rho^{0} \partial_{s} u_{1} \partial_{t} \varphi \mathrm{e}^{\mathrm{i} \lambda_{j} s} \Phi\right) \mathrm{d} t \mathrm{~d} x \\
& +\frac{i}{\lambda_{j}} \int_{I} M_{y}\left(a^{0} \partial_{y} u^{1} \frac{\mathrm{d} \Phi}{\mathrm{d} y} \varphi(0, x)\right) \mathrm{d} x-i \lambda_{j} \int_{Q_{T}} M_{s, y}\left(\rho^{1}\left(\partial_{t} u_{0}+\partial_{s} u_{1}\right) \varphi \mathrm{e}^{\mathrm{i} \lambda_{j} s} \Phi\right) \mathrm{d} t \mathrm{~d} x \\
& +\int_{Q_{T}} M_{s, y}\left(a^{0} \partial_{y} u_{1} \partial_{x} \varphi \mathrm{e}^{\mathrm{i} \lambda_{j} s} \Phi\right) \mathrm{d} t \mathrm{~d} x-\int_{Q_{T}} M_{s, y}\left(a^{0} \frac{\mathrm{d} \Phi}{\mathrm{d} y} \partial_{x} \varphi u_{1} \mathrm{e}^{\mathrm{i} \lambda_{j} s}\right) \mathrm{d} t \mathrm{~d} x \\
& -\frac{i}{\lambda_{j}} \int_{I} M_{y}\left(a^{0} \frac{\mathrm{d} \Phi}{\mathrm{d} y}\right) \partial_{x} \varphi(0, x) u_{0}(0, x) \mathrm{d} x+\int_{Q_{T}} M_{s, y}\left(a^{1}\left(\partial_{x} u_{0}+\partial_{y} u_{1}\right) \frac{\mathrm{d} \Phi}{\mathrm{d} y} \varphi \mathrm{e}^{\mathrm{i} \lambda_{j} s}\right) \mathrm{d} t \mathrm{~d} x \\
& +\int_{Q_{T}} M_{s, y}\left(B \cdot\left(\nabla_{t, x} u_{0}+\nabla_{s, y} u_{1}\right) \varphi \mathrm{e}^{\mathrm{i} \lambda_{j} s} \Phi\right) \mathrm{d} t \mathrm{~d} x=\int_{Q_{T}} M_{s, y}\left(f \varphi \mathrm{e}^{\mathrm{i} \lambda_{j} s} \Phi\right) \mathrm{d} t \mathrm{~d} x,
\end{aligned}
$$

which combined to (3.14) allows us to prove (see [10] for more details) that $u_{0}, u_{1}$ satisfy the variational equation

$$
\left\{\begin{array}{l}
\int_{Q_{T}} M_{s, y}\left(-2 \rho^{0} \partial_{s} u_{1} \partial_{t} \psi+a^{0} \partial_{y} u_{1} \partial_{x} \psi-a^{0} \partial_{x y}^{2} \psi u_{1}\right) \mathrm{d} t \mathrm{~d} x \\
+\int_{Q_{T}} M_{s, y}\left(-\rho^{1}\left(\partial_{t} u_{0}+\partial_{s} u_{1}\right) \partial_{s} \psi+a^{1}\left(\partial_{x} u_{0}+\partial_{y} u_{1}\right) \partial_{y} \psi\right) \mathrm{d} t \mathrm{~d} x \\
+\int_{Q_{T}} M_{s, y}\left(B \cdot\left(\nabla_{t, x} u_{0}+\nabla_{s, y} u_{1}\right) \psi\right) \mathrm{d} t \mathrm{~d} x=\int_{Q_{T}} M_{s, y}(f \psi) \mathrm{d} t \mathrm{~d} x, \quad \forall \psi \in H_{0}^{1}\left(Q_{T} ; \mathcal{W}^{1}\right)
\end{array}\right.
$$

and the initial conditions (3.13).

Contrarily to [10] where the wave equation was considered in the whole space $\mathbb{R}^{N}$, problem (3.1) is stated in a bounded interval with boundary conditions. Thus, besides of equations (3.14), (4.7) and the initial conditions (3.13) we need to obtain some boundary conditions for $u_{1}$ in order to determine the functions $u_{0}, u_{1}$. This is the main novelty with respect to Theorem 3.5 in [10] and it is carried out in the next step.

Step 2. Let us extend (4.7) to more general functions $\psi$ which do not necessarily vanish in $x=\alpha, x=\beta$. Since we consider functions $\psi$ from $Q_{T}$ into $\mathcal{W}^{1}$, they are a linear combination of functions of the type $g_{j}(t, x, y) \mathrm{e}^{\mathrm{i} \lambda_{j} s}$, 
with $g_{j}(t, x,.) \in W_{j}$. By linearity it is enough to assume that $\psi$ is just of the form $\psi=g_{j}(t, x, y) \mathrm{e}^{\mathrm{i} \lambda_{j} s}$ for a particular $j$. We distinguish the cases $j \in J_{1}$ or $j \in J_{2}$ and we take special functions $g_{j}$ in every case.

- $j \in J_{1}$ : By (3.14) (see Rem. 3.9), we can decompose $u_{1}$ as $\hat{u}_{1}+\tilde{u}_{1}$, where $\hat{u}_{1}$ does not depend on $s$ and $\tilde{u}_{1} \in L^{\infty}\left(0, T ; L^{2}\left(I ; \mathcal{W}^{1}\right)\right)$ can be written as

$$
\tilde{u}_{1}(t, x, s, y)=\sum_{l \in \mathbb{Z} \backslash\{0\}} h_{l}(t, x, y) \mathrm{e}^{\mathrm{i} \lambda_{l} s}
$$

for some functions $h_{l} \in L^{\infty}\left(0, T ; L^{2}\left(I ; W_{l}\right)\right)$ satisfying

$$
\underset{t \in(0, T)}{\operatorname{supess}} \sum_{l \in \mathbb{Z} \backslash\{0\}} \lambda_{l}^{2} \int_{I \times Y}\left|h_{l}\right|^{2} \mathrm{~d} x \mathrm{~d} y<+\infty .
$$

Then, taking in (4.7) $\psi=\psi(t, x, s, y)=\varphi(t, x) \Phi(y) \mathrm{e}^{-\mathrm{i} \lambda_{j} s}$, with $\varphi \in H_{0}^{1}\left(Q_{T}\right), \Phi \in W_{j}, \Phi \not \equiv 0$ and using (4.8), and $M_{s}\left(\mathrm{e}^{\mathrm{i} p s}\right)=0$ if $p \neq 0, M_{s}\left(\mathrm{e}^{\mathrm{i} p s}\right)=1$ if $p=0$, we have

$$
\begin{aligned}
\int_{Q_{T}} M_{s, y}\left(a^{0} \partial_{y} u_{1} \partial_{x} \psi-\partial_{x}\left(a^{0} \partial_{y} \psi\right) u_{1}\right) \mathrm{d} t \mathrm{~d} x= & \int_{Q_{T}} \partial_{x} \varphi M_{y}\left(a^{0} \partial_{y} \hat{u}_{1} \Phi-a^{0} \partial_{y} \Phi \hat{u}_{1}\right) M_{s}\left(\mathrm{e}^{\mathrm{i} \lambda_{j} s}\right) \mathrm{d} t \mathrm{~d} x \\
& +\sum_{l \in \mathbb{Z} \backslash\{0\}} \int_{Q_{T}} \partial_{x} \varphi M_{y}\left(a^{0} \partial_{y} h_{l} \Phi-a^{0} \partial_{y} \Phi h_{l}\right) M_{s}\left(\mathrm{e}^{\mathrm{i}\left(\lambda_{l}-\lambda_{j}\right) s}\right) \mathrm{d} t \mathrm{~d} x \\
& =\int_{Q_{T}} \partial_{x} \varphi M_{y}\left(a^{0}\left(\partial_{y} h_{j} \Phi-h_{j} \frac{\mathrm{d} \Phi}{\mathrm{d} y}\right)\right) \mathrm{d} t \mathrm{~d} x,
\end{aligned}
$$

but since $\operatorname{dim}\left(W_{j}\right)=1$ the function $h_{j}$ satisfies $h_{j}(t, x, y)=c_{j}(t, x) \Phi(y)$, with $c_{j} \in L^{\infty}\left(0, T ; L^{2}(I)\right)$ and thus

$$
\int_{Q_{T}} \partial_{x} \varphi M_{y}\left(a^{0}\left(\partial_{y} h_{j} \Phi-h_{j} \frac{\mathrm{d} \Phi}{\mathrm{d} y}\right)\right) \mathrm{d} t \mathrm{~d} x=0 .
$$

So, for such $\psi$, equation (4.7) reduces to

$$
\left\{\begin{array}{l}
\int_{Q_{T}} M_{s, y}\left(-2 \rho^{0} \partial_{s} u_{1} \partial_{t} \psi-\rho^{1}\left(\partial_{t} u_{0}+\partial_{s} u_{1}\right) \partial_{s} \psi+a^{1}\left(\partial_{x} u_{0}+\partial_{y} u_{1}\right) \partial_{y} \psi\right) \mathrm{d} t \mathrm{~d} x \\
+\int_{Q_{T}} M_{s, y}\left(B \cdot\left(\nabla_{t, x} u_{0}+\nabla_{s, y} u_{1}\right) \psi\right) \mathrm{d} t \mathrm{~d} x=\int_{Q_{T}} M_{s, y}(f \psi) \mathrm{d} t \mathrm{~d} x
\end{array}\right.
$$

By the density of $H_{0}^{1}(I)$ into $L^{2}(I)$, this equation holds true for $\psi(t, x, s, y)=\varphi(t, x) \Phi(y) \mathrm{e}^{-\mathrm{i} \lambda_{j} s}$, with $\varphi \in$ $H_{0}^{1}\left(0, T ; L^{2}(I)\right)$ and not only in $H_{0}^{1}\left(Q_{T}\right)$. Reasoning then by linearity and density, we deduce (3.15).

- $j \in J_{2}$ : Since $\operatorname{dim}\left(W_{j}\right)=2$, we can consider a basis $\{\Phi, \Psi\}$ of $W_{j}$, such that

$$
\begin{cases}a^{0}\left(\alpha^{*}\right) \frac{\mathrm{d} \Phi}{\mathrm{d} y}\left(\alpha^{*}\right)=0, a^{0}\left(\alpha^{*}\right) \frac{\mathrm{d} \Psi}{\mathrm{d} y}\left(\alpha^{*}\right) \neq 0 & \text { if } c_{\alpha} \neq 0 \\ \Phi\left(\alpha^{*}\right)=0, \Psi\left(\alpha^{*}\right) \neq 0 & \text { if } c_{\alpha}=0 .\end{cases}
$$

For $\varphi \in H^{1}\left(Q_{T}\right)$ such that $\varphi_{\mid t=0}=\varphi_{\mid t=T}=\varphi_{\mid x=\beta}=0$ we define

$$
v_{\varepsilon}(t, x)=\varphi(t, x)\left(\Phi\left(\frac{x}{\varepsilon}\right)-\mu_{\varepsilon} \Psi\left(\frac{x}{\varepsilon}\right)\right) \mathrm{e}^{\mathrm{i} \lambda_{j} \frac{t}{\varepsilon}},
$$


with

$$
\mu_{\varepsilon}=\left\{\begin{array}{llrl}
\frac{\mathrm{d} \Phi}{\mathrm{d} y}\left(\frac{\alpha}{\varepsilon}\right) / \frac{\mathrm{d} \Psi}{\mathrm{d} y}\left(\frac{\alpha}{\varepsilon}\right) & \text { if } & c_{\alpha} & \neq 0 \\
\Phi\left(\frac{\alpha}{\varepsilon}\right) / \Psi\left(\frac{\alpha}{\varepsilon}\right) & \text { if } & c_{\alpha}=0 .
\end{array}\right.
$$

Observe that thanks to $\Phi, \Psi, a^{0} \frac{\mathrm{d} \Phi}{\mathrm{d} y}, a^{0} \frac{\mathrm{d} \Psi}{\mathrm{d} y}$ continuous and (3.6), we have that $\mu_{\varepsilon}$ is well defined for $\varepsilon$ small enough and satisfies

$$
\mu_{\varepsilon} \rightarrow 0
$$

Taking $v_{\varepsilon}$ as test function in (3.1), we get

$$
\begin{aligned}
& -\int_{Q_{T}} \rho_{\varepsilon} \partial_{t} u_{\varepsilon} \partial_{t} \varphi \mathrm{e}^{\mathrm{i} \lambda_{j} \frac{t}{\varepsilon}} \Phi\left(\frac{x}{\varepsilon}\right) \mathrm{d} t \mathrm{~d} x-\frac{i \lambda_{j}}{\varepsilon} \int_{Q_{T}} \rho_{\varepsilon} \partial_{t} u_{\varepsilon} \varphi \mathrm{e}^{\mathrm{i} \lambda_{j} \frac{t}{\varepsilon}}\left(\Phi\left(\frac{x}{\varepsilon}\right)-\mu_{\varepsilon} \Psi\left(\frac{x}{\varepsilon}\right)\right) \mathrm{d} t \mathrm{~d} x \\
& +\frac{d_{\alpha}}{c_{\alpha}} \int_{\{x=\alpha\}} u_{\varepsilon} \varphi \mathrm{e}^{\mathrm{i} \lambda_{j} \frac{t}{\varepsilon}}\left(\Phi\left(\frac{\alpha}{\varepsilon}\right)-\mu_{\varepsilon} \Psi\left(\frac{\alpha}{\varepsilon}\right)\right) \mathrm{d} t+\int_{Q_{T}} a_{\varepsilon} \partial_{x} u_{\varepsilon} \partial_{x} \varphi \mathrm{e}^{\mathrm{i} \lambda_{j} \frac{t}{\varepsilon}} \Phi\left(\frac{x}{\varepsilon}\right) \mathrm{d} t \mathrm{~d} x \\
& +\frac{1}{\varepsilon} \int_{Q_{T}} a_{\varepsilon} \partial_{x} u_{\varepsilon}\left(\frac{\mathrm{d} \Phi}{\mathrm{d} y}\left(\frac{x}{\varepsilon}\right)-\mu_{\varepsilon} \frac{\mathrm{d} \Psi}{\mathrm{d} y}\left(\frac{x}{\varepsilon}\right)\right) \varphi \mathrm{e}^{\mathrm{i} \lambda_{j} \frac{t}{\varepsilon}} \mathrm{d} t \mathrm{~d} x+\int_{Q_{T}} B_{\varepsilon} \cdot \nabla_{t, x} u_{\varepsilon} \varphi \mathrm{e}^{\mathrm{i} \lambda_{j} \frac{t}{\varepsilon}} \Phi\left(\frac{x}{\varepsilon}\right) \mathrm{d} t \mathrm{~d} x \\
& =\int_{Q_{T}} f_{\varepsilon} \varphi \mathrm{e}^{\mathrm{i} \lambda_{j} \frac{t}{\varepsilon}} \Phi\left(\frac{x}{\varepsilon}\right) \mathrm{d} t \mathrm{~d} x+O_{\varepsilon},
\end{aligned}
$$

where $O_{\varepsilon}$ is due to (4.10) and where the third term is defined as zero in the case where $c_{\alpha}=0$ because by definition of $\mu_{\varepsilon}$, we have $\Phi\left(\frac{\alpha}{\varepsilon}\right)-\mu_{\varepsilon} \Psi\left(\frac{\alpha}{\varepsilon}\right)=0$ if $c_{\alpha}=0$.

Let us pass to the limit in each term of this equality. The calculus is similar to the one in the proof of Theorem 3.5 in [10] but simplified thanks to the absence of terms at $t=0$. We prefer to explicit the calculus because in [10] the corresponding sequence $v_{\varepsilon}$ has compact support and then there is not problem in the integrations by parts, while here we must use the special structure of the test functions $v_{\varepsilon}$ defined above.

First term. Using the expression $(2.11)$ of $\rho_{\varepsilon},(4.5)$ and $M_{s}\left(\mathrm{e}^{\mathrm{i} \lambda_{j} s}\right)=0$, we get

$$
-\int_{Q_{T}} \rho_{\varepsilon} \partial_{t} u_{\varepsilon} \partial_{t} \varphi \mathrm{e}^{\mathrm{i} \lambda_{j} \frac{t}{\varepsilon}} \Phi\left(\frac{x}{\varepsilon}\right) \mathrm{d} t \mathrm{~d} x=-\int_{Q_{T}} M_{s, y}\left(\rho^{0} \partial_{s} u_{1} \partial_{t} \varphi \mathrm{e}^{\mathrm{i} \lambda_{j} s} \Phi\right) \mathrm{d} t \mathrm{~d} x+O_{\varepsilon} .
$$

Second term. Using the expression (2.11) of $\rho_{\varepsilon}$, integrating by parts with respect to $t$ the term containg $\rho^{0}$, and taking into account $\varphi_{\mid t=0}=0,(4.10)$ and (4.5), we have

$$
\begin{aligned}
-\frac{i \lambda_{j}}{\varepsilon} \int_{Q_{T}} \rho_{\varepsilon} \partial_{t} u_{\varepsilon} \mathrm{e}^{\mathrm{i} \lambda_{j} \frac{t}{\varepsilon}} \varphi\left(\Phi\left(\frac{x}{\varepsilon}\right)-\mu_{\varepsilon} \Psi\left(\frac{x}{\varepsilon}\right)\right) \mathrm{d} t \mathrm{~d} x= & \frac{i \lambda_{j}}{\varepsilon} \int_{Q_{T}} \rho^{0}\left(\frac{x}{\varepsilon}\right) u_{\varepsilon} \partial_{t} \varphi \mathrm{e}^{\mathrm{i} \lambda_{j} \frac{t}{\varepsilon}}\left(\Phi\left(\frac{x}{\varepsilon}\right)-\mu_{\varepsilon} \Psi\left(\frac{x}{\varepsilon}\right)\right) \mathrm{d} t \mathrm{~d} x \\
& -\frac{\lambda_{j}^{2}}{\varepsilon^{2}} \int_{Q_{T}} \rho^{0}\left(\frac{x}{\varepsilon}\right) u_{\varepsilon} \varphi \mathrm{e}^{\mathrm{i} \lambda_{j} \frac{t}{\varepsilon}}\left(\Phi\left(\frac{x}{\varepsilon}\right)-\mu_{\varepsilon} \Psi\left(\frac{x}{\varepsilon}\right)\right) \mathrm{d} t \mathrm{~d} x \\
& -i \lambda_{j} \int_{Q_{T}} M_{s, y}\left(\rho^{1}\left(\partial_{t} u_{0}+\partial_{s} u_{1}\right) \varphi \mathrm{e}^{\mathrm{i} \lambda_{j} s} \Phi\right) \mathrm{d} t \mathrm{~d} x+O_{\varepsilon} .
\end{aligned}
$$

In the first term on the right-hand side of this equality we use (4.3) with $\zeta_{\varepsilon}=u_{\varepsilon}$, which gives

$$
\begin{aligned}
& \frac{i \lambda_{j}}{\varepsilon} \int_{Q_{T}} \rho^{0}\left(\frac{x}{\varepsilon}\right) u_{\varepsilon} \partial_{t} \varphi \mathrm{e}^{\mathrm{i} \lambda_{j} \frac{t}{\varepsilon}}\left(\Phi\left(\frac{x}{\varepsilon}\right)-\mu_{\varepsilon} \Psi\left(\frac{x}{\varepsilon}\right)\right) \mathrm{d} t \mathrm{~d} x=i \lambda_{j} \int_{Q_{T}} M_{s, y}\left(\rho^{0} u_{1} \partial_{t} \varphi \mathrm{e}^{\mathrm{i} \lambda_{j} s} \Phi\right) \mathrm{d} t \mathrm{~d} x+O_{\varepsilon} \\
& =\int_{Q_{T}} M_{s, y}\left(\rho^{0} u_{1} \partial_{t} \varphi \partial_{s} e^{\mathrm{i} \lambda_{j} s} \Phi\right) \mathrm{d} t \mathrm{~d} x+O_{\varepsilon}=-\int_{Q_{T}} M_{s, y}\left(\rho^{0} \partial_{s} u_{1} \partial_{t} \varphi \mathrm{e}^{\mathrm{i} \lambda_{j} s} \Phi\right) \mathrm{d} t \mathrm{~d} x+O_{\varepsilon} .
\end{aligned}
$$


Thus, we have proved

$$
\begin{aligned}
-\frac{i \lambda_{j}}{\varepsilon} \int_{Q_{T}} \rho_{\varepsilon} \partial_{t} u_{\varepsilon} \varphi \mathrm{e}^{\mathrm{i} \lambda_{j} \frac{t}{\varepsilon}}\left(\Phi\left(\frac{x}{\varepsilon}\right)-\mu_{\varepsilon} \Psi\left(\frac{x}{\varepsilon}\right)\right) \mathrm{d} t \mathrm{~d} x= & -\int_{Q_{T}} M_{s, y}\left(\rho^{0} \partial_{s} u_{1} \partial_{t} \varphi \mathrm{e}^{\mathrm{i} \lambda_{j} s} \Phi\right) \mathrm{d} t \mathrm{~d} x \\
& -\frac{\lambda_{j}^{2}}{\varepsilon^{2}} \int_{Q_{T}} \rho^{0}\left(\frac{x}{\varepsilon}\right) u_{\varepsilon} \varphi \mathrm{e}^{\mathrm{i} \lambda_{j} \frac{t}{\varepsilon}}\left(\Phi\left(\frac{x}{\varepsilon}\right)-\mu_{\varepsilon} \Psi\left(\frac{x}{\varepsilon}\right)\right) \mathrm{d} t \mathrm{~d} x \\
& -i \lambda_{j} \int_{Q_{T}} M_{s, y}\left(\rho^{1}\left(\partial_{t} u_{0}+\partial_{s} u_{1}\right) \varphi \mathrm{e}^{\mathrm{i} \lambda_{j} s} \Phi\right) \mathrm{d} t \mathrm{~d} x+O_{\varepsilon} .
\end{aligned}
$$

Third term. Taking into account that thanks to the compact imbedding of the trace operator from $H^{1}\left(Q_{T}\right)$ into $L^{2}(\{x=\alpha\}), u_{\varepsilon}$ converges strongly to $u_{0}$ in $L^{2}((0, T) \times\{\alpha\})$ and that $M_{s}\left(\mathrm{e}^{\mathrm{i} \lambda_{j} s}\right)=0$, we get

$$
\frac{d_{\alpha}}{c_{\alpha}} \int_{\{x=\alpha\}} u_{\varepsilon} \varphi \mathrm{e}^{\mathrm{i} \lambda \lambda_{j} \frac{t}{\varepsilon}}\left(\Phi\left(\frac{\alpha}{\varepsilon}\right)-\mu_{\varepsilon} \Psi\left(\frac{\alpha}{\varepsilon}\right)\right) \mathrm{d} t=\frac{d_{\alpha}}{c_{\alpha}} \int_{\{x=\alpha\}} u_{0} \varphi M_{y, s}\left(\mathrm{e}^{\mathrm{i} \lambda_{j} s}\right) \Phi\left(\alpha^{*}\right) \mathrm{d} t+O_{\varepsilon}=O_{\varepsilon} .
$$

Fourth term. From (4.5) and $M_{s}\left(\mathrm{e}^{\mathrm{i} \lambda_{j} s}\right)=0$, we get

$$
\int_{Q_{T}} a_{\varepsilon} \partial_{x} u_{\varepsilon} \partial_{x} \varphi \mathrm{e}^{\mathrm{i} \lambda_{j} \frac{t}{\varepsilon}} \Phi\left(\frac{x}{\varepsilon}\right) \mathrm{d} t \mathrm{~d} x=\int_{Q_{T}} M_{s, y}\left(a^{0} \partial_{y} u_{1} \partial_{x} \varphi \mathrm{e}^{\mathrm{i} \lambda_{j} s} \Phi\right) \mathrm{d} t \mathrm{~d} x+O_{\varepsilon} .
$$

Fifh term. We reason similarly to the second term, i.e. we use expression (2.10) of $a_{\varepsilon}$, then (4.10), then an integration by parts with respect to $x$ in the term containing $a^{0}$ and then (4.5). We get

$$
\begin{aligned}
& \frac{1}{\varepsilon} \int_{Q_{T}} a_{\varepsilon} \partial_{x} u_{\varepsilon}\left(\frac{\mathrm{d} \Phi}{\mathrm{d} y}\left(\frac{x}{\varepsilon}\right)-\mu_{\varepsilon} \frac{\mathrm{d} \Psi}{\mathrm{d} y}\left(\frac{x}{\varepsilon}\right)\right) \varphi \mathrm{e}^{\mathrm{i} \lambda_{j} \frac{t}{\varepsilon}} \mathrm{d} t \mathrm{~d} x \\
= & -\frac{1}{\varepsilon} \int_{\{x=\alpha\}} a^{0}\left(\frac{\alpha}{\varepsilon}\right) u_{\varepsilon}\left(\frac{\mathrm{d} \Phi}{\mathrm{d} y}\left(\frac{\alpha}{\varepsilon}\right)-\mu_{\varepsilon} \frac{\mathrm{d} \Psi}{\mathrm{d} y}\left(\frac{\alpha}{\varepsilon}\right)\right) \varphi \mathrm{e}^{\mathrm{i} \lambda_{j} \frac{t}{\varepsilon}} \mathrm{d} t \\
& -\frac{1}{\varepsilon} \int_{Q_{T}} a^{0}\left(\frac{x}{\varepsilon}\right) u_{\varepsilon}\left(\frac{\mathrm{d} \Phi}{\mathrm{d} y}\left(\frac{x}{\varepsilon}\right)-\mu_{\varepsilon} \frac{\mathrm{d} \Psi}{\mathrm{d} y}\left(\frac{x}{\varepsilon}\right)\right) \partial_{x} \varphi \mathrm{e}^{\mathrm{i} \lambda_{j} \frac{t}{\varepsilon}} \mathrm{d} t \mathrm{~d} x \\
& -\frac{1}{\varepsilon^{2}} \int_{Q_{T}} u_{\varepsilon} \frac{\mathrm{d}}{\mathrm{d} y}\left(a^{0}\left(\frac{x}{\varepsilon}\right)\left(\frac{\mathrm{d} \Phi}{\mathrm{d} y}\left(\frac{x}{\varepsilon}\right)-\mu_{\varepsilon} \frac{\mathrm{d} \Psi}{\mathrm{d} y}\left(\frac{x}{\varepsilon}\right)\right)\right) \varphi \mathrm{e}^{\mathrm{i} \lambda_{j} \frac{t}{\varepsilon}} \mathrm{d} t \mathrm{~d} x \\
& +\int_{Q_{T}} M_{s, y}\left(a^{1}\left(\partial_{x} u_{0}+\partial_{y} u_{1}\right) \frac{\mathrm{d} \Phi}{\mathrm{d} y} \varphi \mathrm{e}^{\mathrm{i} \lambda_{j} s}\right) \mathrm{d} t \mathrm{~d} x+O_{\varepsilon},
\end{aligned}
$$

where the first term on the right-hand side vanishes due to

$$
u_{\varepsilon \mid x=\alpha}=0 \text { if } c_{\alpha}=0, \quad \frac{\mathrm{d} \Phi}{\mathrm{d} y}\left(\frac{\alpha}{\varepsilon}\right)-\mu_{\varepsilon} \frac{\mathrm{d} \Psi}{\mathrm{d} y}\left(\frac{\alpha}{\varepsilon}\right)=0 \text { if } c_{\alpha} \neq 0
$$

and the second term can be estimated using (4.3) with $\zeta_{\varepsilon}=u_{\varepsilon}$ and (4.10), which gives

$$
-\frac{1}{\varepsilon} \int_{Q_{T}} a^{0}\left(\frac{x}{\varepsilon}\right) u_{\varepsilon}\left(\frac{\mathrm{d} \Phi}{\mathrm{d} y}\left(\frac{x}{\varepsilon}\right)-\mu_{\varepsilon} \frac{\mathrm{d} \Psi}{\mathrm{d} y}\left(\frac{x}{\varepsilon}\right)\right) \partial_{x} \varphi \mathrm{e}^{\mathrm{i} \lambda_{j} \frac{t}{\varepsilon}} \mathrm{d} t \mathrm{~d} x=-\int_{Q_{T}} M_{s, y}\left(a^{0} \frac{\mathrm{d} \Phi}{\mathrm{d} y} \partial_{x} \varphi u_{1} \mathrm{e}^{\mathrm{i} \lambda_{j} s}\right) \mathrm{d} t \mathrm{~d} x+O_{\varepsilon} .
$$

Thus, we have proved

$$
\begin{aligned}
& \frac{1}{\varepsilon} \int_{Q_{T}} a_{\varepsilon} \partial_{x} u_{\varepsilon}\left(\frac{\mathrm{d} \Phi}{\mathrm{d} y}\left(\frac{x}{\varepsilon}\right)-\mu_{\varepsilon} \frac{\mathrm{d} \Psi}{\mathrm{d} y}\left(\frac{x}{\varepsilon}\right)\right) \varphi \mathrm{e}^{\mathrm{i} \lambda_{j} \frac{t}{\varepsilon}} \mathrm{d} t \mathrm{~d} x=-\int_{Q_{T}} M_{s, y}\left(a^{0} \frac{\mathrm{d} \Phi}{\mathrm{d} y} \partial_{x} \varphi u_{1} \mathrm{e}^{\mathrm{i} \lambda_{j} s}\right) \mathrm{d} t \mathrm{~d} x \\
& -\frac{1}{\varepsilon^{2}} \int_{Q_{T}} u_{\varepsilon} \frac{\mathrm{d}}{\mathrm{d} y}\left(a^{0}\left(\frac{x}{\varepsilon}\right)\left(\frac{\mathrm{d} \Phi}{\mathrm{d} y}\left(\frac{x}{\varepsilon}\right)-\mu_{\varepsilon} \frac{\mathrm{d} \Psi}{\mathrm{d} y}\left(\frac{x}{\varepsilon}\right)\right)\right) \varphi \mathrm{e}^{\mathrm{i} \lambda_{j} \frac{t}{\varepsilon}} \mathrm{d} t \mathrm{~d} x \\
& +\int_{Q_{T}} M_{s, y}\left(a^{1}\left(\partial_{x} u_{0}+\partial_{y} u_{1}\right) \frac{\mathrm{d} \Phi}{\mathrm{d} y} \varphi \mathrm{e}^{\mathrm{i} \lambda_{j} s}\right) \mathrm{d} t \mathrm{~d} x+O_{\varepsilon} .
\end{aligned}
$$


Sixth and seventh terms. Using the expression of $B_{\varepsilon}$ given in (2.12), the two-scale convergence of $f_{\varepsilon}$ to $f$ and $M_{s}\left(\mathrm{e}^{\mathrm{i} \lambda_{j} s}\right)=0$, we have

$$
\begin{gathered}
\int_{Q_{T}} B_{\varepsilon} \cdot \nabla_{t, x} u_{\varepsilon} \varphi \mathrm{e}^{\mathrm{i} \lambda_{j} \frac{t}{\varepsilon}} \Phi\left(\frac{x}{\varepsilon}\right) \mathrm{d} t \mathrm{~d} x=\int_{Q_{T}} M_{s, y}\left(B \cdot\left(\nabla_{t, x} u_{0}+\nabla_{s, y} u_{1}\right) \varphi \mathrm{e}^{\mathrm{i} \lambda_{j} s} \Phi\right) \mathrm{d} t \mathrm{~d} x+O_{\varepsilon} . \\
\int_{Q_{T}} f_{\varepsilon} \varphi \mathrm{e}^{\mathrm{i} \lambda_{j} \frac{t}{\varepsilon}} \Phi\left(\frac{x}{\varepsilon}\right) \mathrm{d} t \mathrm{~d} x=\int_{Q_{T}} M_{s, y}\left(f \varphi \mathrm{e}^{\mathrm{i} \lambda_{j} s} \Phi\right) \mathrm{d} t \mathrm{~d} x+O_{\varepsilon} .
\end{gathered}
$$

Taking into account the estimates obtained for the different terms of (4.11) and

$$
-\lambda_{j}^{2} \rho^{0}\left(\frac{x}{\varepsilon}\right)\left(\Phi\left(\frac{x}{\varepsilon}\right)-\mu_{\varepsilon} \Psi\left(\frac{x}{\varepsilon}\right)\right)-\frac{\mathrm{d}}{\mathrm{d} y}\left(a^{0}\left(\frac{x}{\varepsilon}\right)\left(\frac{\mathrm{d} \Phi}{\mathrm{d} y}\left(\frac{x}{\varepsilon}\right)-\mu_{\varepsilon} \frac{\mathrm{d} \Psi}{\mathrm{d} y}\left(\frac{x}{\varepsilon}\right)\right)\right)=0 \text { a.e. in } Q_{T},
$$

(which follows from $\Phi$ and $\Psi$ in $W_{j}$ ) we have then proved that (4.7) holds for $\psi(t, x, s, y)=\varphi(t, x) \mathrm{e}^{\mathrm{i} \lambda_{j} s} \Phi(y)$ with $\varphi \in H^{1}\left(Q_{T}\right), \varphi_{\mid t=0}=\varphi_{\mid t=T}=\varphi_{\mid x=\beta}=0$, and $\Phi \in W_{j}$, satisfying

$$
a^{0}\left(\alpha^{*}\right) \frac{\mathrm{d} \Phi}{\mathrm{d} y}\left(\alpha^{*}\right)=0 \text { if } c_{\alpha} \neq 0, \quad \Phi\left(\alpha^{*}\right)=0 \text { if } c_{\alpha}=0
$$

A similar argument proves that (4.7) also holds for $\psi(t, x, s, y)=\varphi(t, x) \mathrm{e}^{\mathrm{i} \lambda_{j} s} \Phi(y)$ with $\Phi \in W_{j}$, such that

$$
a^{0}\left(\beta^{*}\right) \frac{\mathrm{d} \Phi}{\mathrm{d} y}\left(\beta^{*}\right)=0 \text { if } c_{\beta} \neq 0, \quad \Phi\left(\beta^{*}\right)=0 \text { if } c_{\beta}=0
$$

and $\varphi \in H^{1}\left(Q_{T}\right)$ such that $\varphi_{\mid t=0}=\varphi_{\mid t=T}=\varphi_{\mid x=\alpha}=0$.

Reasoning by linearity and density, we have then proved (3.16).

Step 3. We have shown that $u_{0}, u_{1}$ satisfy (3.13), (3.14), (3.15) and (3.16). To finish the proof of Theorem 3.4, it remains to show that this system has a unique solution. Using the decomposition (3.21) of $u_{1}$ and taking into account that, thanks to $\tilde{u}_{1} \in L^{\infty}\left(0, T ; L^{2}\left(I ; \mathcal{W}^{1}\right)\right)$, to know $\partial_{s} u_{1}, \partial_{y} u_{1}$ at $t=0$ and $s=0$ is equivalent to know $u_{1}$ at $t=0$, we get that the uniqueness is an immediate consequence of Theorem 4.2 below. The proof is not given here because it is similar to the one of Theorem Appendix B.1 in [10].

Theorem 4.2. For $I=(\alpha, \beta) \subset \mathbb{R}$, four real functions $\rho^{0} \in L_{\sharp}^{\infty}(Y), a^{0} \in L_{\sharp}^{\infty}(Y), \rho^{1} \in L^{\infty}\left(Q_{T} ; C_{\sharp}(\mathbb{R} \times Y)\right)$, $a^{1} \in L^{\infty}\left(Q_{T} ; C_{\sharp}(\mathbb{R} \times Y)\right)$, satisfying $(2.2)$ and $(2.9)$, and a function $B \in L^{\infty}\left(Q_{T} ; C_{\sharp}(\mathbb{R} \times Y)\right)^{2}$. Then, for every $g \in L^{1}\left(0, T ; L^{2}\left(I ; L_{\sharp}^{2}(\mathbb{R} \times Y)\right)\right)$ and every $w^{0} \in L^{2}\left(I ; \mathcal{W}^{1}\right)$, there exists a unique solution $w \in L^{1}\left(0, T ; L^{2}\left(I ; \mathcal{W}^{1}\right)\right)$ of the variational problem

$$
\begin{gathered}
w_{\mid t=0}=w^{0} \\
\left\{\begin{array}{l}
\int_{Q_{T}} M_{s, y}\left(-2 \rho^{0} \partial_{s} w \partial_{t} \psi-\rho^{1} \partial_{s} w \partial_{s} \psi+a^{1} \partial_{y} w \partial_{y} \psi+B \cdot \nabla_{s, y} w \psi \mathrm{d} t \mathrm{~d} x\right. \\
=\int_{Q_{T}} M_{s, y}(g \psi) \mathrm{d} t \mathrm{~d} x, \quad \forall \psi \in \mathcal{V}_{1}
\end{array}\right. \\
\left\{\begin{array}{l}
\int_{Q_{T}} M_{s, y}\left(-2 \rho^{0} \partial_{s} w \partial_{t} \psi+a^{0} \partial_{y} w \partial_{x} \psi-a^{0} \partial_{x y}^{2} \psi w-\rho^{1} \partial_{s} w \partial_{s} \psi+a^{1} \partial_{y} w \partial_{y} \psi\right) \mathrm{d} t \mathrm{~d} x \\
+\int_{Q_{T}} M_{s, y}\left(B \cdot \nabla_{s, y} w \psi\right) \mathrm{d} t \mathrm{~d} x=\int_{Q_{T}} M_{s, y}(g \psi) \mathrm{d} t \mathrm{~d} x, \quad \forall \psi \in \mathcal{V}_{2} .
\end{array}\right.
\end{gathered}
$$

Moreover, this solution $w$ is in $L^{\infty}\left(0, T ; L^{2}\left(I ; \mathcal{W}^{1}\right)\right)$ and satisfies

$$
\|w\|_{L^{\infty}\left(0, T ; L^{2}\left(I ; \mathcal{W}^{1}\right)\right)} \leq C\left(\left\|w^{0}\right\|_{L^{2}\left(I ; \mathcal{W}^{1}\right)}+\|g\|_{L^{1}\left(0, T ; L^{2}\left(I ; L_{\sharp}^{2}(Y \times \mathbb{R})\right)\right)}\right),
$$

where the constant $C$ only depends on the ellipticity constant of $a^{0}$ and $\rho^{0},\|B\|_{L^{\infty}\left(Q_{T} ; C_{\sharp}(\mathbb{R} \times Y)^{2}\right)}$, $\left\|\partial_{s} \rho^{1}\right\|_{L^{\infty}\left(Q_{T} ; L_{\sharp}^{\infty}(\mathbb{R} \times Y)\right)},\left\|\partial_{s} a^{1}\right\|_{L^{\infty}\left(Q_{T} ; L_{\sharp}^{\infty}(\mathbb{R} \times Y)\right)}$ and $T$. 
Proof of Proposition 3.12. We take $j \in J_{2}$ and two independent eigenfunctions $\Phi_{1}, \Phi_{2} \in W_{j}$ such that

$$
a^{0}\left(\alpha^{*}\right) \frac{\mathrm{d} \Phi_{1}}{\mathrm{~d} y}\left(\alpha^{*}\right)=0 \text { if } c_{\alpha} \neq 0, \quad \Phi_{1}\left(\alpha^{*}\right)=0 \text { if } c_{\alpha}=0, \quad a^{0}\left(\Phi_{1} \frac{\mathrm{d} \Phi_{2}}{\mathrm{~d} y}-\Phi_{2} \frac{\mathrm{d} \Phi_{1}}{\mathrm{~d} y}\right)=1 \text { in } \mathbb{R} .
$$

Then, we define $h \in L^{\infty}\left(0, T ; L^{2}\left(I ; W_{j}\right)\right)$ as the component of $\tilde{u}_{1}$ corresponding to $\mathrm{e}^{\mathrm{i} \lambda_{j} s}$, when we write it as a Fourier sum with respect to the basis $\mathrm{e}^{\mathrm{i} \lambda_{l} s}, l \in \mathbb{Z} \backslash\{0\}$, i.e. $h$ is defined by

$$
h=M_{s}\left(\tilde{u}_{1} \mathrm{e}^{-\mathrm{i} \lambda_{j} s}\right), \text { a.e. in } Q_{T} \times Y .
$$

Since $\Phi_{1}, \Phi_{2}$ are a basis of $W_{j}$, the function $h$ can be decomposed as

$$
h(t, x, y)=h_{1}(t, x) \Phi_{1}(y)+h_{2}(t, x) \Phi_{2}(y), \quad \text { a.e. }(t, x, y) \in Q_{T} \times Y .
$$

Now, for $g \in H^{1}\left(Q_{T}\right), g_{\mid t=0}=g_{\mid t=T}=0, g_{\mid x=\beta}=0$, we define $\psi$ by

$$
\psi(t, x, s, y)=g(t, x) \mathrm{e}^{-\mathrm{i} \lambda_{j} s} \Phi_{1}(y)
$$

and we observe that the decomposition (3.21) of $u_{1}$, with $\hat{u}_{1}$ independent of $s$, and definitions (4.21), (4.22) of $h, h_{1}$ and $h_{2}$, imply

$$
\begin{aligned}
& \int_{Q_{T}} M_{s, y}\left(a^{0} \partial_{y} u_{1} \partial_{x} \psi-a^{0} \partial_{x y}^{2} \psi u_{1}\right) \mathrm{d} t \mathrm{~d} x \\
& =\int_{Q_{T}} M_{y}\left(a^{0}\left(h_{1} \frac{\mathrm{d} \Phi_{1}}{\mathrm{~d} y}+h_{2} \frac{\mathrm{d} \Phi_{2}}{\mathrm{~d} y}\right) \partial_{x} g \Phi_{1}-a^{0}\left(h_{1}\left(\Phi_{1}+h_{2} \Phi_{2}\right) \partial_{x} g \frac{\mathrm{d} \Phi_{1}}{\mathrm{~d} y}\right) \mathrm{d} t \mathrm{~d} x=\int_{Q_{T}} h_{2} \partial_{x} g \mathrm{~d} t \mathrm{~d} x .\right.
\end{aligned}
$$

Therefore, using this function $\psi$ as test function in (3.16), we deduce

$$
\int_{Q_{T}} p \partial_{t} g \mathrm{~d} t \mathrm{~d} x+\int_{Q_{T}} h_{2} \partial_{x} g \mathrm{~d} t \mathrm{~d} x=-\int_{Q_{T}} q g \mathrm{~d} x \mathrm{~d} t, \forall g \in H^{1}\left(Q_{T}\right), g_{\mid t=0}=g_{\mid t=T}=0, g_{\mid x=\beta}=0,
$$

with

$$
\begin{gathered}
p=M_{s, y}\left(-2 \rho^{0} \partial_{s} u_{1} \mathrm{e}^{-\mathrm{i} \lambda_{j} s} \Phi_{1}\right), \\
q=M_{s, y}\left(\mathrm{e}^{-\mathrm{i} \lambda_{j} s}\left(i \lambda_{j} \rho^{1}\left(\partial_{t} u_{0}+\partial_{s} u_{1}\right) \Phi_{1}+a^{1}\left(\partial_{x} u_{0}+\partial_{y} u_{1}\right) \frac{\mathrm{d} \Phi_{1}}{\mathrm{~d} y}\right)\right) \\
+M_{s, y}\left(\left(B \cdot\left(\nabla_{t, x} u_{0}+\nabla_{s, y} u_{1}\right)-f\right) \mathrm{e}^{-\mathrm{i} \lambda_{j} s} \Phi_{1}\right) .
\end{gathered}
$$

Since in (4.23), we have not imposed any boundary condition to $g$ at $x=\alpha$, this is equivalent to

$$
\partial_{t} p+\partial_{x} h_{2}=q, \quad h_{2 \mid x=\alpha}=0 .
$$

By (4.22), and the properties of $\Phi_{1}$, we then deduce

$$
\left(a^{0} \partial_{y} h\right)_{\mid x=\alpha, y=\alpha^{*}}=0 \text { if } c_{\alpha} \neq 0, \quad h_{\mid x=\alpha, y=\alpha^{*}}=0 \text { if } c_{\alpha}=0 .
$$

Since this holds for every Fourier component of $\tilde{u}_{1}$ relative to a Fourier node $\lambda_{j}$, with $j \in J_{2}$ we get

$$
\left(a^{0} \partial_{y} P_{2} \tilde{u}_{1}\right)_{\mid x=\alpha, y=\alpha^{*}}=0 \text { if } c_{\alpha} \neq 0, \quad P_{2} \tilde{u}_{1 \mid x=\alpha, y=\alpha^{*}}=0 \text { if } c_{\alpha}=0 .
$$

A similar reasoning shows

$$
\left(a^{0} \partial_{y} P_{2} \tilde{u}_{1}\right)_{\mid x=\beta, y=\beta^{*}}=0 \text { if } c_{\beta} \neq 0, \quad P_{2} \tilde{u}_{1 \mid x=\beta, y=\beta^{*}}=0 \text { if } c_{\beta}=0 .
$$


Proof of Theorem 3.15. Taking into account the decomposition $u_{1}=\tilde{u}_{1}+\hat{u}_{1}$ given in Remark 3.9, where $\hat{u}_{1}$ does not depend on $s$ and using that the functions in $\mathcal{W}^{1}$ have mean value zero, equations (3.15) and (3.16), with $\rho^{1}, a^{1}$ and $B$ vanishing, are reduced to

$$
\begin{gathered}
\int_{Q_{T}} M_{s, y}\left(-2 \rho^{0} \partial_{s} \tilde{u}_{1} \partial_{t} \psi\right) \mathrm{d} t \mathrm{~d} x=\int_{Q_{T}} M_{s, y}(f \psi) \mathrm{d} t \mathrm{~d} x, \quad \forall \psi \in \mathcal{V}_{1} \\
\int_{Q_{T}} M_{s, y}\left(-2 \rho^{0} \partial_{s} \tilde{u}_{1} \partial_{t} \psi+a^{0} \partial_{y} \tilde{u}_{1} \partial_{x} \psi-a^{0} \partial_{x y}^{2} \psi \tilde{u}_{1}\right) \mathrm{d} t \mathrm{~d} x=\int_{Q_{T}} M_{s, y}(f \psi) \mathrm{d} t \mathrm{~d} x, \quad \forall \psi \in \mathcal{V}_{2} .
\end{gathered}
$$

On the other hand, using that $\hat{u}_{1}$ belongs to $L^{\infty}\left(0, T ; L^{2}\left(I ; H_{\sharp}^{1}(Y)\right)\right.$, and that $\tilde{u}_{1}$ belongs to $L^{\infty}\left(0, T ; L^{2}\left(I, \mathcal{W}^{1}\right)\right)$ we deduce that $\hat{u}_{1}$ and $\tilde{u}_{1}$ can be decomposed as in $(3.45),(3.46)$. The problem is now to determine the coefficients in the series defining $\hat{u}_{1}$ and $\tilde{u}_{1}$.

Using as test function in (3.18) $\Phi_{j}$ if $j \in J_{1}^{+}$, or $\Phi_{j l}, l \in\{1,2\}$, if $j \in J_{2}^{+}$we deduce that the coefficient $\hat{u}_{j}$ are given by (3.49) and (3.50).

The determination of the coefficients $\tilde{\mathrm{u}}_{j}$ is more involved. We distinguish the cases $j \in J_{1}, j \in J_{2}$.

- Case $j \in J_{1}$. We take in (4.24), $\psi(t, x, s, y)=\varphi(t, x) \Phi_{j}(y) \mathrm{e}^{-\mathrm{i} \lambda_{j} s}$, with $\varphi \in C_{c}^{\infty}\left(Q_{T}\right)$. This gives

$$
-2 i \lambda_{j} \int_{Q_{T}} \tilde{\mathrm{u}}_{j} \partial_{t} \varphi \mathrm{d} t \mathrm{~d} x=\int_{Q_{T}} f_{j} \varphi \mathrm{d} t \mathrm{~d} x, \quad \forall \varphi \in C_{c}^{\infty}(\Omega)
$$

and then

$$
2 i \lambda_{j} \partial_{t} \tilde{\mathbf{u}}_{j}=f_{j} \text { in } Q_{T} .
$$

To determine $\tilde{u}_{j}$ from this equation we also need an initial condition. This can be obtained from (3.13). Namely, multiplying the second equation in (3.13) by $\rho^{0} \Phi_{j}$, taking the mean value in $y$, and using $(3.36),(3.46)$ and $M_{y}\left(\rho^{0} \Phi_{j}\right)=0$, we have

$$
i \lambda_{j}\left(\tilde{\mathrm{u}}_{j}-\tilde{\mathrm{u}}_{-j}\right)_{\mid t=0}=\vartheta_{j} \text {, a.e. in } I .
$$

Analogously, multiplying the third equation in (3.13) by $a^{0} \partial_{y} \Phi_{j}$, taking the mean value in $y$ and using $u_{1}=\hat{u}_{1}+\tilde{u}_{1}$ combined to (3.35), (3.45) and (3.46), we get

$$
\left(\hat{\mathrm{u}}_{j}+\tilde{\mathrm{u}}_{j}+\tilde{\mathrm{u}}_{-j}\right)_{\mid t=0}=u_{j}^{1} \text {, a.e. in } I .
$$

or taking into account (3.49)

$$
\left(\tilde{\mathbf{u}}_{j}+\tilde{\mathbf{u}}_{-j}\right)_{\mid t=0}=u_{j}^{1}+\frac{m_{j}}{\lambda_{j}^{2}} \partial_{x} u_{0 \mid t=0}, \text { a.e. in } I .
$$

From (4.27) and (4.28) we then get

$$
\begin{aligned}
& \tilde{\mathrm{u}}_{j \mid t=0}=\frac{1}{2}\left(u_{j}^{1}+\frac{m_{j}}{\lambda_{j}^{2}} \partial_{x} u_{0 \mid t=0}-\frac{\vartheta_{j}}{\lambda_{j}} i\right), \text { a.e. in } I, \forall j \in J_{1}^{+} . \\
& \tilde{\mathrm{u}}_{-j \mid t=0}=\frac{1}{2}\left(u_{j}^{1}+\frac{m_{j}}{\lambda_{j}^{2}} \partial_{x} u_{0 \mid t=0}+\frac{\vartheta_{j}}{\lambda_{j}} i\right), \text { a.e. in } I, \forall j \in J_{1}^{+},
\end{aligned}
$$

which combined to (4.26) proves (3.51) and (3.52).

- Case $j \in J_{2}$. As above, taking $\psi(t, x, s, y)=\varphi(t, x) \Phi_{j l}(y) \mathrm{e}^{-\mathrm{i} \lambda_{j} s}, l=1,2, \varphi \in C_{c}^{\infty}\left(Q_{T}\right)$ as test function in (4.25) we deduce (3.53).

Reasoning as in the case $j \in J_{1}$ we also deduce that (3.13) implies (3.55) and (3.56). On the other hand, we recall that by Proposition 3.12, (4.25) implies (3.54).

Acknowledgements. The authors have been partially supported by the project MTM2011-24457 of the "Ministerio de Economía y Competitividad" of Spain. 


\section{REFERENCES}

[1] G. Allaire, Homogenization and two-scale convergence. SIAM J. Math. Anal. 23 (1992) 1482-1518.

[2] G. Allaire and L. Friz, Localization of high-frequence waves propagating in a locally periodic medium. In vol. 140A, Proc. of Roy. Soc. Edinburgh (2010) 897-926.

[3] G. Allaire, M. Palombaro and J. Rauch, Diffractive behavior of the wave equation in periodic media: weak convergence analysis. Ann. Mat. Pura Appl. 188 (2009) 561-589.

[4] A. Bensoussan, J.L. Lions and G. Papanicolau, Asymptotic analysis for periodic structures. North Holland, Amsterdam (1978).

[5] S. Brahim-Otsmane, G.A. Francfort and F. Murat, Correctors for the homogenization of the wave and heat equations. J. Math. Pures Appl. 71 (1992) 197-231.

[6] M. Brassart and M. Lenczner, A two scale model for the periodic homogenization of the wave equation. J. Math. Pure Appl. 93 (2010) 474-517.

[7] J. Casado-Díaz and I. Gayte, A general compactness result and its application the two-scale convergence of almost periodic functions. C. R. Acad. Sci. Paris I 323 (1996) 329-334.

[8] J. Casado-Díaz and I. Gayte, The two-scale convergence method applied to generalized Besicovitch spaces. Proc. Roy. Soc. London A 458 (2002) 2925-2946.

[9] J. Casado-Díaz, J. Couce-Calvo, F. Maestre and J.D. Martín-Gómez, Homogenization and corrector for the wave equation with discontinuos coefficients in time. J. Math. Anal. Appl. 379 (2011) 664-681.

[10] J. Casado-Díaz, J. Couce-Calvo, F. Maestre and J.D. Martín-Gómez, Homogenization and correctors for the wave equation with periodic coefficients. Math. Models Methods Appl. Sci. 24 (2014) 1343-1388.

[11] F. Colombini and S. Spagnolo, On the convergence of solutions of hyperbolic equations. Commun. Partial Differ. Eq. 3 (1978) $77-103$.

[12] G.A. Francfort and F. Murat, Oscillations and energy densities in the wave equation. Commun. Partial Differ. Eq. 17 (1992) $1785-1865$.

[13] F. Murat, H-convergence. Séminaire d'Analyse Fonctionnelle et Numérique, 1977-78. Université d'Alger; F. Murat, L. Tartar. H-convergence. Topics in the Mathematical Modelling of Composite Materials. In vol. 31 of Progr. Nonlin. Differ. Eq. Appl., edited by L. Cherkaev and R.V. Kohn. Birkaüser, Boston (1998) 21-43.

[14] G. Nguetseng, A general convergence result for a functional related to the theory of homogenization. SIAM J. Math. Anal. 20 (1989) 608-623.

[15] G. Nguetseng, Homogenization structures and applications I. Z. Anal. Anwen. 22 (2003) 73-107.

[16] G. Nguetseng, M. Sango and J.L. Woukeng, Reiterated ergodic algebras and applications. Commun. Math. Phys. 300 (2010) $835-876$.

[17] S. Spagnolo, Sulla convergenza di soluzioni di equazioni paraboliche ed ellittiche. Ann. Scuola Norm. Sup. Pisa Cl. Sci. 22 (1968) 571-597.

[18] L. Tartar, The general theory of the homogenization. A personalized introduction. Springer, Berlin Heidelberger (2009). 